\title{
Resonance Zones and Lobe Volumes for Volume-Preserving Maps
}

\author{
H. E. Lomelí and J. D. Meiss * \\ Department of Mathematics \\ Department of Applied Mathematics \\ Instituto Tecnológico Autónomo de México \\ University of Colorado \\ Mexico, DF 01000 \\ Boulder, CO 80309-0526 \\ lomeli@itam.mx \\ James.Meiss@colorado.edu
}

November 8, 2018

\begin{abstract}
We study exact, volume-preserving diffeomorphisms that have heteroclinic connections between a pair of normally hyperbolic invariant manifolds. We develop a general theory of lobes, showing that the lobe volume is given by an integral of a generating form over the primary intersection, a subset of the heteroclinic orbits. Our definition reproduces the classical action formula in the planar, twist map case. For perturbations from a heteroclinic connection, the lobe volume is shown to reduce, to lowest order, to a suitable integral of a Melnikov function.
\end{abstract}

\section{Introduction}

The computation of the volume of incoming and exit sets for a "nearly invariant" region is the first step in the development of a dynamical theory of transport. For area-preserving maps, it is common for these regions to be bounded by segments of the stable and unstable manifolds hyperbolic invariant sets, typically periodic orbits or cantori (Aubry-Mather sets) [MMP84, RKW88. In this case the resulting set is a "resonance zone" [MMP87, Eas91], and its exit and entrance sets are "lobes" of the "turnstile." When the splitting between the manifolds is small, the resonance zone is nearly invariant.

The volume of a lobe is a not only a measure of the separation of the stable and unstable manifolds, it is also the flux of trajectories escaping from the resonance zone. This flux provides an estimate for the escape time from the resonance. It is known that the average exit time from a region is equal to the ratio of accessible volume of the region (the fraction of the volume that can

${ }^{*}$ HL was supported in part by Asociación Mexicana de Cultura. JDM was supported in part by NSF grant DMS0707659 and by the Mathematical Sciences Research Institute in Berkeley. Useful conversations with Holger Dullin, Richard Montgomery and Rafael de la Llave are gratefully acknowledged. 
be reached by orbits from the outside) to the flux [Mac94, Mei97]. Though the accessible volume is difficult to compute it is certainly bounded by the total volume of the region, so the flux provides an upper bound on the average exit time. Moreover, if the flux goes to zero, but the accessible volume does not, then the average exit time must go to infinity.

In this paper, we discuss the construction of lobes for resonance zones of volume-preserving maps. Our goal is to generalize the results of [MMP84] that provide formulas for the lobe areas for the two-dimensional maps and of [Mac94] for the case of three-dimensional, incompressible vector fields. Indeed, one of the three open problems posed by MacKay at the end of [Mac94] is to generalize his flow results to the case of maps.

The theory of [Mac94] applies to exact-symplectic maps. Recall that a map is symplectic when there is a closed two form, $\omega$, (for example $\omega=d q \wedge d p$ ) that is preserved by $f: f^{*} \omega=\omega ! 1$ A map is exact-symplectic when $\omega$ is exact, $\omega=-d \nu$ (for example $\nu=p d q$ ) and there exists a function $S$ defined by

$$
f^{*} \nu-\nu=d S
$$

An often studied case is that of twist maps, which have Lagrangian generating functions - the discrete analogues of the Lagrangians for differential equations Mei92].

Remarkably, the generating function (1) provides a way to compute the area of a lobe (or of the resonance zone itself) in terms of the action, the formal sum of $S$ along an orbit [MMP84, MMP87]. The result is that a two-dimensional integral over a lobe is reduced to a zero-dimensional integral, the difference between the actions of the orbits homoclinic to the hyperbolic invariant set. Thus to compute the lobe area one only needs to find these homoclinic orbits and carry out the sum; this is considerably easier than constructing the entire lobe boundary and computing the two-dimensional integral. Moreover, since the action is stationary on the orbits, its computation is second-order accurate.

For the case of incompressible vector fields, a similar result also holds. On the manifold $M=\mathbb{R}^{3}$, when $\nabla \cdot u=0$ there exists a vector potential $A$ so that $u=\nabla \times A$. Here $A$ is more properly thought of as a one-form $\beta=A \cdot d l$ and it gives rise to a variational principle for orbits of the flow. Moreover, MacKay has shown that the one-form $\beta$ can be used to compute the flux through two-dimensional surfaces [Mac94]. More generally, when $\Omega$ is an exact volume form and $u$ is an exact incompressible vector field, then the curl relationship generalizes to the statement that $i_{u} \Omega=d \beta$ is exact and $\beta$ provides the generating form (see $\$ 7.2$ and [LM08]) as well as a variational principle[GM03.

In this paper we study exact volume-preserving maps. For such maps there is also an analogue of (1) in which the generator $S$ becomes a differential form $\lambda$, see $\$ 2$, Such forms were implicitly described by [Car04] in the context of constructing implicit generating functions for volume-preserving maps [LM08. We will primarily study the three-dimensional case where the generator $\lambda$ is a one-form.

\footnotetext{
${ }^{1}$ We recall the notation for the pullback, $f^{*}$, and similar concepts in the Appendix.
} 
We will construct a resonance zone based on a pair of normally hyperbolic invariant manifolds, say $A$ and $B$. For the three-dimensional case, the natural objects are periodic orbits and invariant circles. The boundary of the resonance will consist of pieces of the codimension-one stable $W^{\mathrm{s}}(A)$ and unstable $W^{\mathrm{u}}(B)$ manifolds of these invariant sets. The exit and incoming sets are obtained by iterating the resonance, see 4 .

Unlike the two-dimensional case, the boundary of a resonance does not always consist solely of pieces of stable and unstable manifolds. Indeed for this to happen, the manifolds would have to intersect on a "proper boundary", see $\$ 4.2$. However, we have observed that the set of heteroclinic intersections commonly contains components that are themselves bi-asymptotic to the invariant sets; these preclude the existence of heteroclinic proper boundaries [LM00]. A similar phenomena occurs for three-dimensional volume-preserving flows: when the two-dimensional manifolds of saddle equilibria intersect, they typically do so only along a few "primary" heteroclinic orbits Bro81, Hol84, Mac94. To remedy this, a "cap" must be used to complete the resonance boundary. Even though the cap is - to a large extent - arbitrary, this choice does not change the volume of the exit and incoming lobes of the resonance.

Our main result is Thm. 4 in $\$ 5$, which states that the volume of the exit and incoming lobes is given by

$$
\operatorname{Vol}(\mathcal{E})=\operatorname{Vol}(\mathcal{I})=\sum_{k \in \mathbb{Z}} \int_{\eta}\left(f^{k}\right)^{*} \lambda=\int_{\mathcal{P}(A, B)} \lambda
$$

Here the set $\mathcal{P}(A, B)=\bigcup_{k \in \mathbb{Z}} f^{k}(\eta)$ consists of the primary heteroclinic intersections of the manifolds $W^{\mathrm{s}}(A)$ and $W^{\mathrm{u}}(B)$, and $\eta$ is the restriction of $\mathcal{P}(A, B)$ to a fundamental domain. Thus to compute the volume of $n$-dimensional lobes one needs only to do $(n-2)$-dimensional integrals along submanifolds of heteroclinic intersections.

For the case of nearly-integrable systems, a widely used technique for detecting such heteroclinic intersections is the Poincaré-Melnikov method [Mel63, HM82, LMRR08]. Indeed, this method detects - at first order - precisely the primary intersections.

The classical Melnikov function computes the rate at which the distance between the manifolds changes with a perturbation, say $\delta$. For two-dimensional maps, an integral of the Melnikov function between a pair of zeros gives the rate of change of the flux with $\delta$ [MM88]; a similar result also holds for incompressible vector fields [Mac94]. We will show in $\$ 6$ that this same results holds for volume-preserving maps.

We conclude by presenting several examples and applications in $\S 7$. 


\section{Exact Volume-preserving maps}

\subsection{Definition}

Let $M$ be an $n$-dimensional manifold. A volume form $\Omega$ is a nondegenerate $n$-form on $M$. A map $f: M \rightarrow M$ on an $n$-dimensional manifold preserves the volume form $\Omega$ if

$$
f^{*} \Omega=\Omega .
$$

For example if $M=\mathbb{R}^{n}$, and $\Omega=d x_{1} \wedge d x_{2} \wedge \ldots \wedge d x_{n}$, then $f$ is volume preserving when its Jacobian has unit determinant everywhere, $\operatorname{det}(D f)=1$. Suppose now that $\Omega$ is exact, i.e., there exists an $(n-1)$-form $\alpha$ such that $\Omega=d \alpha$. By analogy with the symplectic case (1), we can also define exact volume-preserving maps.

Definition 1 (Exact Volume Preserving). A diffeomorphism $f: M \rightarrow M$ is exact-volume preserving if there exists an $(n-1)$-form $\alpha$ such that $d \alpha=\Omega$ and a generating $(n-2)$-form $\lambda$ such that

$$
f^{*} \alpha-\alpha=d \lambda
$$

It is clear that if $f$ is exact-volume preserving, then $f^{-1}$ is also. Moreover, if $f=g_{1} \circ g_{2}$ is the composition of exact volume-preserving maps with generating forms $\lambda_{1}$ and $\lambda_{2}$, respectively, then since $\left(g_{1} \circ g_{2}\right)^{*}=g_{2}^{*} \circ g_{1}^{*}$,

$$
f^{*} \alpha-\alpha=g_{2}^{*}\left(g_{1}^{*} \alpha-\alpha\right)+g_{2}^{*} \alpha-\alpha=d\left(g_{2}^{*} \lambda_{1}+\lambda_{2}\right) .
$$

Thus $f$ is exact-volume preserving with $\lambda=g_{2}^{*} \lambda_{1}+\lambda_{2}$. Consequently, the set of exact volumepreserving maps is a subgroup of the group of volume-preserving diffeomorphisms, we denote it $\operatorname{Diff}_{\alpha}(M)$. We discuss some of the consequences of exactness in [LM08].

As an application that we will need later, consider the composition of an exact volume-preserving map with itself. In this case we can use a telescoping sum to conclude

$$
\left(f^{n}\right)^{*} \alpha-\alpha=\sum_{j=0}^{n-1}\left(\left(f^{j+1}\right)^{*} \alpha-\left(f^{j}\right)^{*} \alpha\right)=\sum_{j=0}^{n-1}\left(f^{j}\right)^{*}\left(f^{*} \alpha-\alpha\right)=\sum_{j=0}^{n-1} d\left(\left(f^{j}\right)^{*} \lambda\right) .
$$

Thus $f^{n}$ is exact-volume preserving with the form

$$
\lambda_{n}=\sum_{j=0}^{n-1}\left(f^{j}\right)^{*} \lambda
$$




\subsection{Geometrical Implications}

Exact volume-preserving maps arise naturally in the context of perturbations from integrable maps. For example suppose that $f$ is a map on the phase space $M=\mathbb{T}^{d} \times \mathbb{R}$ with volume form $\Omega=$ $d z \wedge d \theta_{1} \wedge \ldots \wedge d \theta_{d}$. This form is exact with $\alpha=z \wedge d \theta_{1} \wedge \ldots \wedge d \theta_{d}$. One integrable map on $M$ is

$$
f(\theta, z)=(\theta+\rho(z), z)
$$

with the rotation vector $\rho: \mathbb{R} \rightarrow \mathbb{T}^{d}$. This map is also exact volume preserving: if we define the vector field $W(\theta, z)=\left(0, \int z \rho^{\prime} d z\right)$, then $f^{*} \alpha-\alpha=d \lambda$ where

$$
\lambda=i_{W} d \theta_{1} \wedge \ldots \wedge d \theta_{d}
$$

The invariant tori of this map are "rotational" tori, and as we argue next, any volume preserving map on $M$ with a rotational invariant torus must be exact.

A rotational torus on $\mathbb{T}^{d} \times \mathbb{R}$ is a $d$-dimensional torus that is homotopic to the zero section $\left\{(\theta, 0): \theta \in \mathbb{T}^{d}\right\}$. The net flux crossing a rotational torus $\mathcal{T}$ is the difference between the signed volume below $f(\mathcal{T})$ and that below $\mathcal{T}$ :

$$
\operatorname{Flux}(\mathcal{T})=\int_{\mathcal{T}} f^{*} \alpha-\alpha .
$$

Note that if $f$ is volume preserving, and $\mathcal{T}$ and $\hat{\mathcal{T}}$ are any two rotational tori then the volume contained between them, $\Delta V=\int_{\hat{\mathcal{T}}} \alpha-\int_{\mathcal{T}} \alpha$, is invariant. This implies that $\operatorname{Flux}(\mathcal{T})$ is independent of the choice of torus.

Thus, if $\operatorname{Flux}(\mathcal{T})=0$ then $f(\mathcal{T}) \cap \mathcal{T} \neq \emptyset$ for any rotational torus; this intersection property is used in the generalization of KAM theory for exact volume-preserving maps [Xia92]. Conversely, if $f$ has an invariant rotational torus then $\operatorname{Flux}(\mathcal{T})=0$ by definition. Consequently if $f$ has a rotational invariant torus then it is necessarily exact.

Exactness can be used to simplify the computation of the volume of certain regions. Suppose that $M$ is any orientable manifold, and $\mathcal{R}$ is a region whose boundary can be decomposed into pieces that are related by iteration, $\mathcal{S}$ and $f(\mathcal{S})$, and such that $\mathcal{S} \cap f(\mathcal{S})=\mathcal{C}$ is an invariant codimension-two submanifold. We assign orientations to $\mathcal{R}$ and $\mathcal{S}$, and by iteration to $f(\mathcal{S})$; thus

$$
\partial \mathcal{R}=f(\mathcal{S})-\mathcal{S},
$$

and $\partial \mathcal{S}=\mathcal{C}$. Then, when $f$ is exact, 2 immediately gives

$$
\operatorname{Vol}(\mathcal{R})=\int_{\mathcal{R}} \Omega=\int_{\mathcal{S}} f^{*} \alpha-\alpha=\int_{\mathcal{C}} \lambda
$$

Thus we can compute the volume of $\mathcal{R}$ simply by integrating over $\mathcal{C}$. This formula is closely related to those that we will use to compute the volume of exit and incoming sets in $\$ 5$. 


\subsection{Examples}

Since every closed form on $M=\mathbb{R}^{n}$ is exact, the volume form $\Omega=d x_{1} \wedge \ldots \wedge d x_{n}$ is exact with, for example, $\alpha=x_{1} d x_{2} \wedge \ldots \wedge d x_{n}$. If $f$ is volume preserving, then $f^{*} \alpha-\alpha$ is a closed $(n-1)$-form, but since every closed form on $\mathbb{R}^{n}$ is exact, $f$ is exact.

However, exactness is not automatic on more general manifolds. For example, a perturbation of (4) is the one-action map $f$ on $M=\mathbb{T}^{d}, \times \mathbb{R}$

$$
f(\theta, z)=(\theta+\rho(z+F(\theta)), z+F(\theta))
$$

for a "force" $F: \mathbb{T}^{d} \rightarrow \mathbb{R}$ is always volume-preserving, but is only exact when the form $F d \theta_{1} \wedge \ldots \wedge d \theta_{d}$ is exact [LM08], or equivalently when

$$
\int_{\mathbb{T}^{d}} F d \theta_{1} \wedge \ldots \wedge d \theta_{d}=0
$$

For example, if $d=2, \rho(z)=\left(z, z^{2}\right)$ and $F=a \cos \theta_{1}+b \cos \theta_{2}+c \cos \left(\theta_{1}+\theta_{2}\right)$ then $(6)$ is generated by

$$
\lambda=\left(\frac{1}{2} Z^{2}+a \sin \theta_{1}\right) d \theta_{2}-\left(\frac{2}{3} Z^{3}+b \sin \theta_{2}+c \sin \left(\theta_{1}+\theta_{2}\right)\right) d \theta_{1},
$$

where $Z=z+F(\theta)$.

In [LM08] we showed that any exact-symplectic map (1) is also exact-volume preserving. For example, reinterpreting (6) as a map on $(\theta, z) \in \mathbb{T}^{2} \times \mathbb{R}^{2}$, then $f$ becomes a "generalized standard" or "Froeschlé" map [Fro72]. It is symplectic with the two-form $\omega=\sum_{i} d \theta_{i} \wedge d z_{i}$ provided that $\rho(z)=\nabla K(z)$ for "kinetic energy" $K$ and $F=-\nabla V(\theta)$ for "potential energy" $V$. Letting $\nu=z \cdot d \theta$, then $f$ is also exact-symplectic and has the generator

$$
S(\theta, z)=Z \cdot \nabla K(Z)-K(Z)-V(\theta)
$$

which is equivalent to the implicit, Lagrangian generating function [Mei92]. For example when $\rho(z)=z$, then $K(z)=\frac{1}{2}|z|^{2}$ and the generator becomes

$$
S(\theta, z)=\frac{1}{2}|Z|^{2}-V(\theta) .
$$

Now setting $\alpha=\frac{1}{2} \nu \wedge \omega$, then $d \alpha=\Omega=d \theta_{1} \wedge d \theta_{2} \wedge d z_{1} \wedge d z_{2}$ and we find that the generator (2) for the generalized standard map is the two-form

$$
\lambda=\frac{1}{2} S(\theta, z) \omega
$$




\section{Normally hyperbolic invariant manifolds}

Resonance zones are most naturally associated with normally hyperbolic invariant manifolds; in the simplest case, with a hyperbolic periodic orbit. In addition to periodic orbits, we will also consider normally hyperbolic invariant circles, but many of our results apply more generally. Here we recall the definition of normal hyperbolicity, and prove a lemma about convergence of forms on the stable manifolds of such sets.

Suppose and $f: M \rightarrow M$ is a diffeomorphism on an $n$-dimensional smooth manifold $M$ and $A$ is a compact invariant set of $f$. We recall one standard definition of normal hyperbolicity.

Definition 2 (Normal Hyperbolicity [DdlLS08]). A compact invariant set $A$ is $r$-normally hyperbolic for $r \in \mathbb{N}$, if there exists an invariant splitting of the tangent bundle $T_{A} M=E^{\mathrm{s}} \oplus E^{\mathrm{u}} \oplus T A$, a Riemannian structure, and positive constants $C, \lambda$ and $\mu$, such that for all $a \in A$,

a) $0<\lambda<\mu^{-r}<1$;

b) $\left\|D f^{t}(a) v\right\| \leq C \lambda^{n}\|v\|$, for all $v \in E_{a}^{\mathrm{s}}$ and $t \in \mathbb{N}$;

c) $\left\|D f^{-t}(a) v\right\| \leq C \lambda^{n}\|v\|$, for all $v \in E_{a}^{\mathrm{u}}$ and $t \in \mathbb{N}$;

d) $\left\|D f^{t}(a) v\right\| \leq C \mu^{|n|}\|v\|$, for all $v \in T_{a} A$ and $t \in \mathbb{Z}$.

The stable manifold theorem applies to $r$-normally hyperbolic sets: there exist $C^{r}$ immersed submanifolds $W^{\mathrm{s}}(A, f)=W^{\mathrm{s}}(A)$, tangent to $E^{\mathrm{s}} \oplus T A$ at $A$, and $W^{\mathrm{u}}(A, f)=W^{\mathrm{u}}(A)$, tangent to $E^{\mathrm{u}} \oplus T A$ at $A$ HPS77a].

We next prove a lemma that we will need in the following sections to show that some of the sums converge. This lemma applies to differential $\ell$-forms on a compact part of a stable or unstable manifold of a normally hyperbolic invariant set.

Let $\Lambda^{\ell}(M)$ denote the linear space of $\ell$-forms on $M$. For any compact set $P \subset M$, there is a natural norm on $\Lambda^{\ell}(P)$ given by:

$$
\|\omega\|_{P}=\sup \left\{\left|\omega_{p}\left(v_{1}, v_{2}, \ldots, v_{\ell}\right)\right|: p \in P, v_{i} \in T_{p} P,\left\|v_{i}\right\|=1, i=1, \ldots, \ell\right\} .
$$

The main point is that for any $p \in P$ and any set of vectors $v_{i} \in T_{p} P$,

$$
\left|\omega_{x}\left(v_{1}, v_{2}, \ldots, v_{\ell}\right)\right| \leq\|\omega\|_{P}\left\|v_{1}\right\|\left\|v_{2}\right\| \cdots\left\|v_{\ell}\right\| .
$$

Definition 3 (Regular Form). A differential $\ell$-form $\omega$ defined on $W^{\mathrm{s}}(A)$ is regular if

$$
\lim _{k \rightarrow \infty}\left(f^{*}\right)^{k} \omega=0 .
$$

Similarly if $\omega$ is defined on $W^{\mathrm{u}}(A)$ then it is regular if

$$
\lim _{k \rightarrow-\infty}\left(f^{*}\right)^{k} \omega=0 \text {. }
$$


In $\$ 5$ and 86 , we will use the following simple consequences of this definition.

Lemma 1. If $\omega$ is regular on $W^{\mathrm{s}}(A)$, then

a) if $P \subset W^{\mathrm{s}}(A)$ is a compact submanifold of dimension $\ell$ then

$$
\lim _{t \rightarrow \infty} \int_{P}\left(f^{t}\right)^{*} \omega=0
$$

b) If $Y$ is a vector field on $W^{\mathrm{s}}(A)$ and $Q \subset W^{\mathrm{s}}(A)$ is submanifold of dimension $\ell-1$ then

$$
\lim _{t \rightarrow \infty} \int_{Q} i_{Y}\left(f^{t}\right)^{*} \omega=0
$$

An $\ell$-form is automatically regular if $\ell$ is large enough so that every independent set of $\ell$ vectors $v_{i} \in T W^{\mathrm{s}}(A)$ is guaranteed to contain sufficiently many vectors in the stable, as opposed to center, directions.

Lemma 2. If $A$ is an $r$-normally hyperbolic invariant set with $\operatorname{dim}(A)=n_{A}$, then every differential $\ell$-form with $\ell>0$ and

$$
\ell \geq n_{A}\left(1+\frac{1}{r}\right)
$$

is regular on any compact subset $P \subset W^{\mathrm{s}}(A)$.

Proof. By hypothesis there is a splitting $T_{A} M=E^{\mathrm{s}} \oplus E^{\mathrm{u}} \oplus T A$ and positive constants $C, \lambda$ and $\mu$ as in Defn. 2. The stable manifold theorem implies that there exists a neighborhood $\mathcal{N}$ of $A$ in $W^{\mathrm{s}}(A)$ and coordinates $\phi=(a, s): \mathcal{N} \rightarrow A \times \mathbb{R}^{n_{s}}$, where $n_{s}=\operatorname{dim}\left(E^{\mathrm{s}}\right)$, such that $s(\xi)=0$ if and only if $\xi \in A$, and such that $\tilde{f}=\phi \circ f \circ \phi^{-1}$ takes the form

$$
\tilde{f}(a, s)=(g(a), L(a) s+r(a, s))
$$

with $r(a, 0)=0, \partial_{2} r(a, 0)=0$ and $L(a)$ is a matrix. In addition, there is an induced Riemannian structure on $\mathcal{N}$ such that $D \tilde{f}$ satisfies the conditions of normal hyperbolicity on the zero section, for some constants $\tilde{C}, \tilde{\lambda}$ and $\tilde{\mu}$, such that $\lambda \leq \tilde{\lambda}<1,1<\tilde{\mu} \leq \mu$, and $\tilde{\mu}^{r} \tilde{\lambda}<1$.

Thus if $P$ is a compact submanifold of $W^{\mathrm{s}}(A)$, the normal hyperbolicity conditions imply that for all $t \in \mathbb{N}$ there exists $m_{1}, m_{2} \in \mathbb{N}$ such that

$$
\left\|\left(f^{t}\right)^{*} \omega\right\|_{P} \leq \tilde{C}\left(\tilde{\mu}^{m_{1}} \tilde{\lambda}^{m_{2}}\right)^{t}\|\omega\|_{P} .
$$

where $m_{1}+m_{2}=\ell$ and $m_{1} \leq n_{A}$. The $r^{t h}$ power of the contraction factor in this equation satisfies

$$
\left(\tilde{\mu}^{m_{1}} \tilde{\lambda}^{m_{2}}\right)^{r}=\left(\tilde{\mu}^{r} \tilde{\lambda}\right)^{m_{1}} \tilde{\lambda}^{\left(m_{2} r-m_{1}\right)}
$$


and (7) implies that

$$
m_{2} r-m_{1}=\left(\ell-m_{1}\right) r-m_{1} \geq\left(\ell-n_{A}\right) r-n_{A} \geq 0 .
$$

Thus both integers $m_{1}$ and $m_{2} r-m_{1}$ are nonnegative. If at least one of these integers is positive, then $\tilde{\mu}^{m_{1}} \tilde{\lambda}^{m_{2}}<1$. If $m_{1}>0$ then all is well. Alternatively, if $m_{1}=0$, then $m_{2} r-m_{1}=\ell r$, which is positive since $\ell>0$ by hypothesis. Consequently, in either case, $\left\|\left(f^{n}\right)^{*} \omega\right\|_{P} \rightarrow 0$ as required.

Remark. It is important to notice that we have to include the manifold $A$ in $W^{\mathrm{s}}(A)$, otherwise we could have invariant forms on $W^{\mathrm{s}}(A)$ that don't satisfy the conclusion of Lem. 2. For instance, in [LM03], an invariant $n-1$ form is constructed on $W^{\mathrm{s}}(A) \backslash A$ when $A$ is a normally hyperbolic invariant circle. We also want to point out that similar computations appear in DdlLS08, and CFdlL05.

If $n_{A}=0$, then Lem. 2 implies that all $\ell$-forms with $\ell \geq 1$ are regular, but if $n_{A}=1$, then $\ell \geq 2$. We will often apply the lemma to the case $\ell=n-1$, in which case an $\ell$-form always is regular on a one-normally hyperbolic invariant manifold when $n_{A} \leq(n-1) / 2$.

\section{Measuring Transport}

In dynamical systems, transport is the study of the motion of collections of trajectories from one region of phase space to another. A natural choice for a region is a subset $\mathcal{R} \subset M$ that is almost invariant in the sense that it consists of a set of points whose orbits belong to $\mathcal{R}$ for a long time. One way to construct such a nearly invariant region is to form its boundary $\partial \mathcal{R}$ (as much as possible) from the invariant manifolds of a pair of normally hyperbolic invariant manifolds; these boundaries are called partial barriers $2^{2}$

For the two-dimensional case this leads to the construction of resonance zones bounded by the invariant manifolds of a periodic orbit or cantorus [MMP84, Eas91]. For a saddle fixed point, $a$, it is natural to base the selection of the exit and incoming sets on primary homoclinic points $p, q \in W^{\mathrm{s}}(a) \cap W^{\mathrm{u}}(a)$, see Fig. 1. In this case the boundary of the resonance is a single partial barrier formed from segments of the stable and unstable manifolds from $a$ to $f(p)$. In other cases, like maps on the cylinder, the resonance zones associated with rotational periodic orbits have upper and lower boundaries, each formed from different branches of stable and unstable manifolds [MMP87.

In this paper we will generalize this construction to the volume-preserving case. To do this, we will not be able to assume that the lobes are bounded entirely by subsets of the invariant manifolds.

In order to restrict the topological possibilities to something manageable, we will usually assume that the phase space $M$ is at most three-dimensional and consider only the simplest hyperbolic invariant sets: fixed points and invariant circles.

\footnotetext{
${ }^{2}$ Another is to use an isolating block [Eas91]
} 


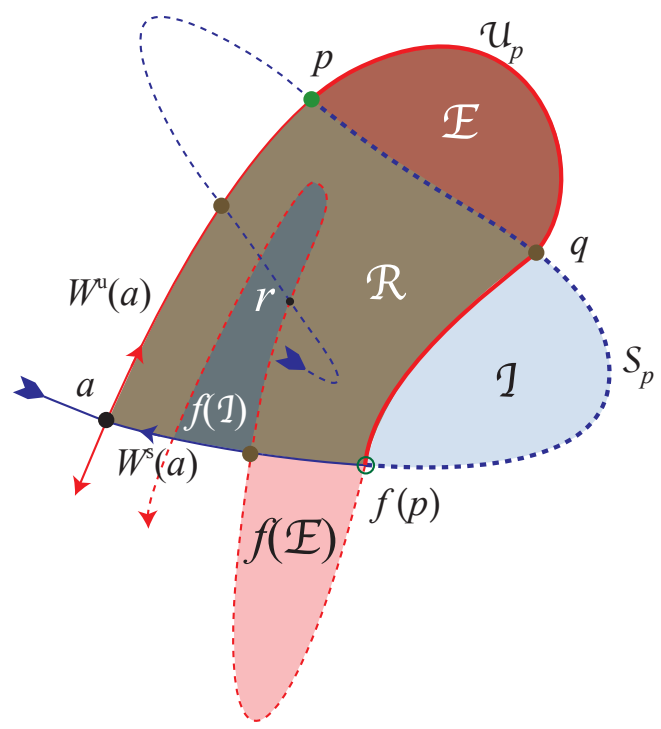

Figure 1: Standard construction of a two-dimensional resonance zone $\mathcal{R}$ for a saddle point $a$, based on a primary homoclinic point $f(p) \in W^{\mathrm{s}}(a) \cap W^{\mathrm{u}}(a)$. The boundary of the resonance zone $\mathcal{R}$ is $W_{f(p)}^{\mathrm{u}}(a) \cup W_{f(p)}^{\mathrm{s}}(a)$ and $\mathcal{R}$ has exit set $\mathcal{E}$ and incoming set $\mathcal{I}$.

\subsection{Incoming and Exit Sets}

Any region $\mathcal{R} \subset M$ has incoming and exit sets. The exit set $\mathcal{E}$ is the set of points in $\mathcal{R}$ that leave in one step: they have forward exit time one. Similarly, the incoming set $\mathcal{I}$ consists of all points not in $\mathcal{R}$ whose images land in $\mathcal{R} !^{3}$

Definition 4 (Incoming and exit sets). The exit and incoming sets for a region $\mathcal{R}$ are

$$
\begin{aligned}
& \mathcal{E}=\mathcal{R} \backslash f^{-1}(\mathcal{R}), \\
& \mathcal{I}=f^{-1}(\mathcal{R}) \backslash \mathcal{R},
\end{aligned}
$$

respectively.

Suppose now that $f$ preserves a measure $\mu$, and that $\mu(\mathcal{R})<\infty$. Since $\mu(A \backslash B)=\mu(A)-$ $\mu(A \cap B)$, then

$$
\begin{aligned}
& \mu(\mathcal{E})=\mu(\mathcal{R})-\mu\left(\mathcal{R} \cap f^{-1}(\mathcal{R})\right), \\
& \mu(\mathcal{I})=\mu\left(f^{-1}(\mathcal{R})\right)-\mu\left(f^{-1}(\mathcal{R}) \cap \mathcal{R}\right) .
\end{aligned}
$$

\footnotetext{
${ }^{3}$ In Mei97, the entry set was defined to be the image of the incoming set and is a subset of $\mathcal{R}$. Here it seems more convenient to use the preimage of the entry set.
} 
My measure preservation, $\mu(\mathcal{R})=\mu\left(f^{-1}(\mathcal{R})\right)$, so that the volume of the exit and incoming sets are equal: $\mu(\mathcal{I})=\mu(\mathcal{E})$.

The incoming and exit sets for a region are often called "lobes" [RKW88, and their union is a "turnstile" [MMP84]. The volume of a lobe - either exit or incoming set - is a simple measure of how fast the points move from $\mathcal{R}$ to its complement. In other words, the lobe volume is a measure of the degree to which a set is invariant. Roughly speaking, if one selects a point at random in $\mathcal{R}$, then it has probability $p=\mu(\mathcal{E}) / \mu(\mathcal{R})$ of landing in $\mathcal{E}$ at each iteration, and so its expected escape time is $1 / p$. This can be made precise [Mei97]: the average time for a trajectory that begins in $\mathcal{I}$ to escape is

$$
\left.\left\langle t_{e x i t}\right\rangle\right|_{\mathcal{I}}=\frac{\mu\left(\mathcal{R}_{a c c}\right)}{\mu(\mathcal{E})},
$$

where $\mathcal{R}_{a c c}$ is the accessible portion of $\mathcal{R}$ : the part that can be reached by trajectories that begin outside $\mathcal{R}$. To obtain more details of the distribution of exit times requires an understanding of the decomposition of $\mathcal{I}$ into regions of fixed exit time [RKW88, Mei97].

\subsection{Fundamental Domains}

To construct the boundaries of a resonance zone, we will use the concepts of fundamental domain and primary intersection of the stable and unstable manifolds of a hyperbolic invariant set [LM00].

In order to form a resonance zone based upon a pair of normally hyperbolic invariant manifolds $A$ and $B$, they must have codimension-one invariant stable and unstable manifolds. To be concrete, we will suppose that

$$
\operatorname{codim}\left(W^{\mathrm{s}}(A)\right)=\operatorname{codim}\left(W^{\mathrm{u}}(B)\right)=1 .
$$

If $\sigma \subset W^{\mathrm{s}}(A)$ is the boundary of a subset of $W^{\mathrm{s}}(A)$ that contains $A$, then we denote the unstable manifold starting at $\sigma$ by $W_{\sigma}^{\mathrm{s}}(A)$ : it is the closed subset of the local stable manifold of $A$ bounded by $\sigma$ :

$$
\sigma=\partial W_{\sigma}^{s}(A)
$$

Similarly, $W_{\gamma}^{\mathrm{u}}(B)$ is the unstable manifold up to $\gamma$ when $\gamma=\partial W_{\gamma}^{\mathrm{u}}(B)$; however, in this case it is convenient to assume that this submanifold is open. Though this definition is not not symmetric, the asymmetry is useful to simplify some proofs.

For our purposes, it will be important that the boundaries of these local manifolds are chosen to be proper. Recall that a neighborhood $N$ of an invariant set $A$ is isolating or a trapping region if $A \subset \operatorname{int}(N)$ and

$$
f(\operatorname{cl}(N)) \subset \operatorname{int}(N) .
$$

Definition 5 (Proper Boundary). Suppose $A$ and $B$ are compact, normally hyperbolic invariant manifolds. A set $\sigma \subset W^{\mathrm{s}}(A)$ is a proper boundary if $W_{\sigma}^{\mathrm{s}}(A)$ is an isolating neighborhood of $A$ in $W^{\mathrm{s}}(A)$. Similarly, $\gamma \subset W^{\mathrm{u}}(B)$ is proper if $W_{\gamma}^{\mathrm{u}}(B)$ is an isolating neighborhood of $B$ for $f^{-1}$. 
It is not hard to see that the stable manifold theorem, e.g. [HPS77b], implies that proper boundaries always exist. These local manifolds behave naturally under iteration:

$$
f\left(W_{\sigma}^{\mathrm{s}}(A)\right)=W_{f(\sigma)}^{\mathrm{s}}(A) \text {, and } f\left(W_{\gamma}^{\mathrm{u}}(B)\right)=W_{f(\gamma)}^{\mathrm{u}}(B) .
$$

Given a proper boundary, an invariant manifold can be partitioned into nonoverlapping fundamental domains that are related by iteration.

Definition 6 (Fundamental Domain). The set $\mathcal{F}^{\mathrm{s}}(A)$ of fundamental domains of $W^{\mathrm{s}}(A)$ is the collection of sets of the form

$$
\mathcal{S}_{\sigma}(A) \equiv W_{\sigma}^{\mathrm{s}}(A) \backslash W_{f(\sigma)}^{\mathrm{s}}(A) .
$$

where $\sigma$ is any proper boundary. Similarly, the set $\mathcal{F}^{\mathrm{u}}(B)$ of fundamental domains in $W^{\mathrm{u}}(B)$ is the collection of sets of the form

$$
\mathcal{U}_{\gamma}(B) \equiv W_{f(\gamma)}^{\mathrm{u}}(B) \backslash W_{\gamma}^{\mathrm{u}}(B),
$$

where $\gamma$ is any proper boundary.

Remark. The closure assumptions imply that $\sigma \subset \mathcal{S}_{\sigma}$ and $\gamma \subset \mathcal{U}_{\gamma}$, but their images are not. In addition, one has $\partial \mathcal{S}_{\sigma}=\sigma \cup f(\sigma)$ and $\partial \mathcal{U}_{\gamma}=\gamma \cup f(\gamma)$.

As an example, consider the two-dimensional map sketched in Fig. 1. In this case, the resonance zone $\mathcal{R}$ is bounded by $W_{f(p)}^{\mathrm{s}}(a) \cup W_{f(p)}^{\mathrm{u}}(a)$ and the fundamental domains $\mathcal{S}_{p}(a)$ and $\mathcal{U}_{p}(a)$ form the boundary of the region $\mathcal{E} \cup \mathcal{I}$, the turnstile for $\mathcal{R}$. For a three-dimensional map, a fundamental domain is an annulus bounded by a proper boundary $\gamma$ and its image $f(\gamma)$.

A consequence of the definition is that the image of a fundamental domain is also a fundamental domain and that $f^{k}\left(\mathcal{S}_{\sigma}\right)=\mathcal{S}_{f^{k}(\sigma)}$ and $f^{k}\left(\mathcal{U}_{\gamma}\right)=\mathcal{U}_{f^{k}(\gamma)}$ for any $k \in \mathbb{Z}$. Moreover, stable and unstable manifolds can be decomposed as the disjoint union of fundamental domains:

$$
\begin{aligned}
W_{\sigma}^{\mathrm{s}}(A) \backslash A & =\bigcup_{t \geq 0} \mathcal{S}_{f^{t}(\sigma)}(A), \\
W_{\gamma}^{\mathrm{u}}(B) \backslash B & =\bigcup_{t<0} \mathcal{U}_{f^{t}(\gamma)}(B) .
\end{aligned}
$$

Consequently, the topology of the intersections of stable and unstable manifolds can be studied

by restricting to appropriate fundamental domains [LM00, LM03. We will use the fundamental domains to construct the incoming and exit sets for a resonance zone.

\subsection{Primary Intersections}

As above, we continue to assume that $f$ has a pair of normally hyperbolic invariant sets $A$ and $B$. In addition, the stable and unstable manifolds $W^{\mathrm{s}}(A)$ and $W^{\mathrm{u}}(B)$ are codimension-one, orientable manifolds as in (9). In addition, we now assume that there is a heteroclinic intersection

$$
W^{\mathrm{s}}(A) \cap W^{\mathrm{u}}(B) \neq \emptyset .
$$


The set of heteroclinic intersections is typically staggeringly complex; in this section we pick out the first or primary intersection to use in the construction of a partial barrier from these manifolds.

For example, consider a pair of saddle fixed points $a$ and $b$, of a two-dimensional map. A point $\eta \in W^{\mathrm{s}}(a) \cap W^{\mathrm{u}}(b)$ is a primary intersection point (p.i.p.) if (recall $W_{\eta}^{\mathrm{u}}(b)$ is open and $W_{\eta}^{\mathrm{s}}(a)$ is closed)

$$
W_{\eta}^{\mathrm{s}}(a) \cap W_{\eta}^{\mathrm{u}}(b)=\emptyset ;
$$

that is, the manifolds up to $\eta$ intersect only at their boundary, Wig92. For example, the points $p$ and $q$ are primary homoclinic points in Fig. 1, but the point $r$ is not since the set $W_{r}^{\mathrm{s}}(a) \cap W_{r}^{\mathrm{u}}(b)$ contains five points. This definition will not work in higher dimensions, since the components of the set $W^{\mathrm{s}}(A) \cap W^{\mathrm{u}}(B)$ need not be proper boundaries even when the intersections are transverse. Instead, we generalize as follows:

Definition 7 (Primary Intersection). A point $\eta \in W^{\mathrm{s}}(A) \cap W^{\mathrm{u}}(B)$ is a primary intersection point if there exist proper boundaries $\gamma$ and $\sigma$ such that

$$
\eta \in W_{\sigma}^{\mathrm{s}}(A) \cap W_{f(\gamma)}^{\mathrm{u}}(B), \text { but } W_{\sigma}^{\mathrm{s}}(A) \cap W_{\gamma}^{\mathrm{u}}(B)=\emptyset .
$$

The set of primary intersections is denoted $\mathcal{P}(A, B)$; it is an invariant set.

For one-dimensional manifolds, this gives the same set as 12 . Primary intersections can also be defined in terms of fundamental domains. Indeed, since $W_{\sigma}^{\mathrm{s}}=\mathcal{S}_{\sigma} \cup W_{f(\sigma)}^{\mathrm{s}}$ and $W_{f(\gamma)}^{\mathrm{u}}=\mathcal{U}_{\gamma} \cup W_{\gamma}^{\mathrm{u}}$ by $(10)$, then if $\eta$ is a primary intersection

$$
\eta \in\left(\mathcal{S}_{\sigma}(A) \cup W_{f(\sigma)}^{\mathrm{s}}(A)\right) \cap\left(\mathcal{U}_{\gamma}(B) \cup W_{\gamma}^{\mathrm{u}}(B)\right)=\mathcal{S}_{\sigma}(A) \cap \mathcal{U}_{\gamma}(B),
$$

since, by $130 \mathcal{S}_{\sigma}(A) \cap W_{\gamma}^{\mathrm{u}}(B)=\mathcal{U}_{\gamma}(B) \cap W_{f(\sigma)}^{\mathrm{s}}(A)=\emptyset$. In addition, 10 and 13p imply that all forward images of the stable fundamental domain are disjoint from the unstable one: $\mathcal{S}_{f^{t}(\sigma)}(A) \cap$ $\mathcal{U}_{\gamma}(B)=\emptyset$ for $t>0$. This means that the intersection index,

$$
\kappa(\mathcal{S}, \mathcal{U}) \equiv \sup \left\{t \in \mathbb{Z}: f^{t}(\mathcal{S}) \cap \mathcal{U} \neq \emptyset\right\}
$$

is zero. Consequently, an alternative characterization of the primary intersection set is [LM00]

$$
\mathcal{P}(A, B)=\left\{\mathcal{S} \cap \mathcal{U}: \kappa(\mathcal{S}, \mathcal{U})=0, \mathcal{S} \in \mathcal{F}^{\mathrm{s}}(A), \mathcal{U} \in \mathcal{F}^{\mathrm{u}}(B)\right\}
$$

We will henceforth make the assumption that the codimension-one manifolds $W^{\mathrm{s}}(A)$ and $W^{\mathrm{u}}(B)$ have transversal primary intersections (the manifolds may still have tangencies elsewhere). In this case, the components of $\mathcal{P}(A, B)$ are codimension-two submanifolds that never cross. For example, in the three-dimensional case, these components must be closed loops or else are curves that are asymptotic to the invariant sets $A$ and $B$. 
If the heteroclinic intersections appear as the result of splitting of separatrices, then typically it is possible to apply Melnikov's method. Recall that there is a correspondence between the zeroes of the Melnikov function and heteroclinic intersections. If all the zeroes are simple then they continue precisely to the set of primary intersections.

Since each fundamental domain generates the entire manifold, we can restrict attention to the set of primary intersections in a particular fundamental domain, say $\mathcal{P} \cap \mathcal{U}_{\gamma}$. A fundamental domain on a two-dimensional manifold is an annulus, but under the natural identification $\gamma \simeq f(\gamma)$ of its boundaries, it can be thought of as a torus $\tilde{\mathcal{U}}_{\gamma}$, see Fig. 2

If $\eta$ is a component of $\mathcal{P}$ then, under this identification, its orbit $f^{t}(\eta)$ is equivalent to a closed loop. Indeed, if $\eta$ is contained in the interior of $\operatorname{cl}\left(\mathcal{U}_{\gamma}\right)$, then it must be a loop since the intersections are assumed transverse. On the other hand, if there is an intersection point $p=\eta \cap \gamma$, then since $\mathcal{P}$ is invariant, the curve $f(\eta) \in \mathcal{P}$ and intersects $f(\gamma)$ at the point $f(p)$. Thus the components $\eta$ and $f(\eta)$ are joined by the natural identification.

Continuing this implies that the full orbit of $p$ lies on $\gamma$ under the identification. This orbit must be finite, since it would otherwise have limit points, violating transversality. Thus, the orbit of $\eta$ becomes a closed loop $\tilde{\eta}$ on $\tilde{\mathcal{U}}_{\gamma}$.

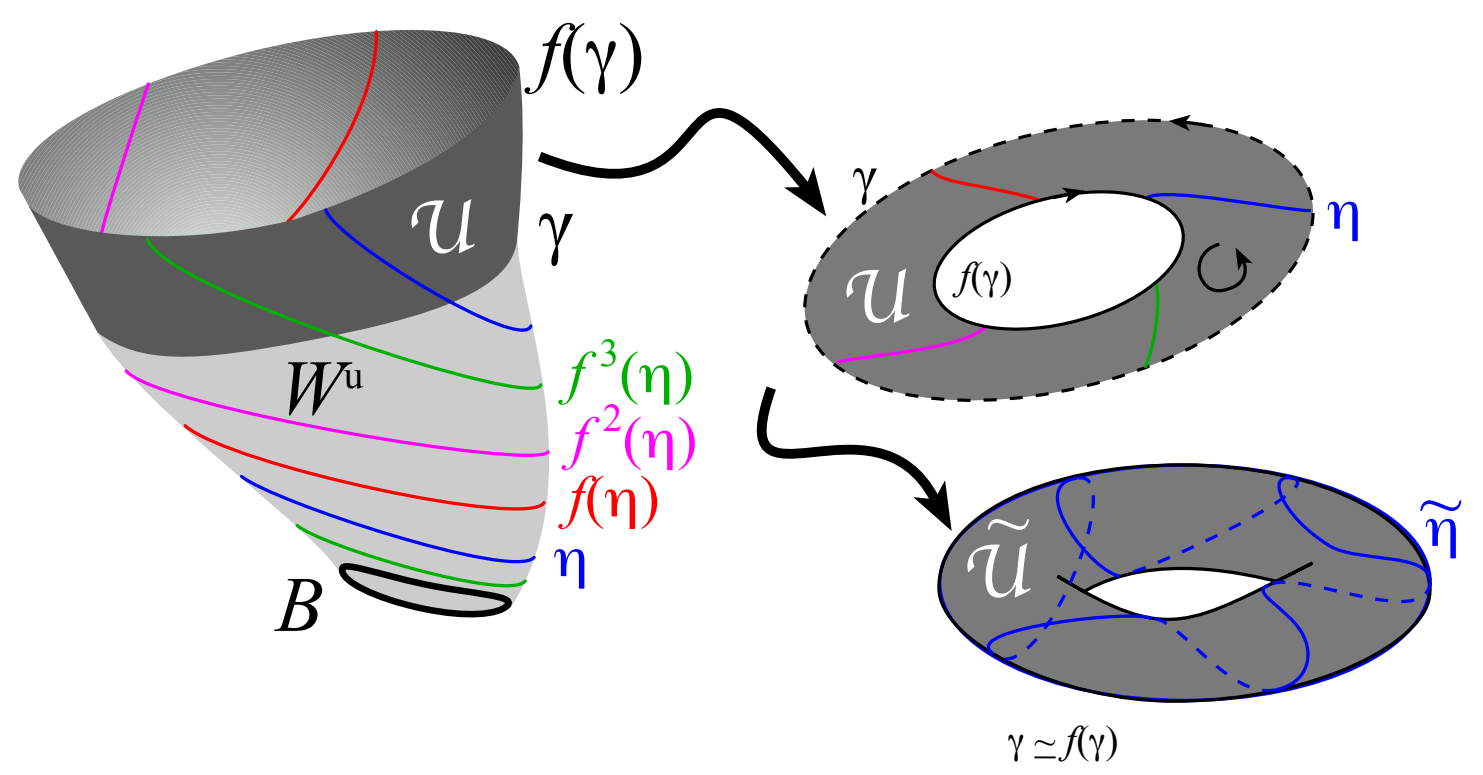

Figure 2: Identifying the boundaries of a fundamental domain gives a torus $\tilde{\mathcal{U}}$ and primary intersection loop $\tilde{\eta}$.

Therefore, on the torus $\tilde{\mathcal{U}}_{\gamma}$, a primary intersection loop can be labeled by its homology class, $(m, n) \in \mathbb{Z}^{2}$. A loop that is homotopic to $\gamma$ will be said to have class $(0,1)$, while loops that correspond to intersections $\eta$ that are asymptotic to $A$ and $B$ will have class $(m, n)$ with $m \neq 0$. 
We previously used this classification to discuss bifurcations of $\mathcal{P}$ that occur when the manifolds develop tangencies as a parameter is varied [LM00, LM03, LRR08].

\subsection{Resonance Zones}

In this section we will establish the basic assumptions to construct resonance zones in terms of codimension one stable and unstable manifolds of normally hyperbolic invariant manifolds.

The geometry of resonance zones based on arbitrary normally hyperbolic invariant sets could be quite complicated. In order to gain some intuition, we start by describing the case that $f$ is a map on a three-dimensional manifold $M$ and assume that $A$ and $B$ are hyperbolic fixed points or invariant circles. Motivated by this discussion, we will then propose a set of geometrical assumptions to define a partial barrier for a resonance zone.

Three typical resonance zones $\mathcal{R}$ for this case are sketched in Fig. 3 . For example, if $A$ and $B$ are fixed points, $\mathcal{R}$ is a ball that is, roughly speaking, bounded by $W_{\sigma}^{\mathrm{s}}(A)$ and $W_{\gamma}^{\mathrm{u}}(B)$ for some proper boundaries $\sigma$ and $\gamma$. The sketch corresponds to the "integrable" case when these manifolds coincide forming a saddle connection from $A$ to $B$. More generally the boundary of $\mathcal{R}$ will be made from a partial barrier $\mathcal{D}$ that is constructed from $W_{\sigma}^{\mathrm{s}}(A)$ and $W_{\gamma}^{\mathrm{u}}(B)$ plus a "cap" (see below).
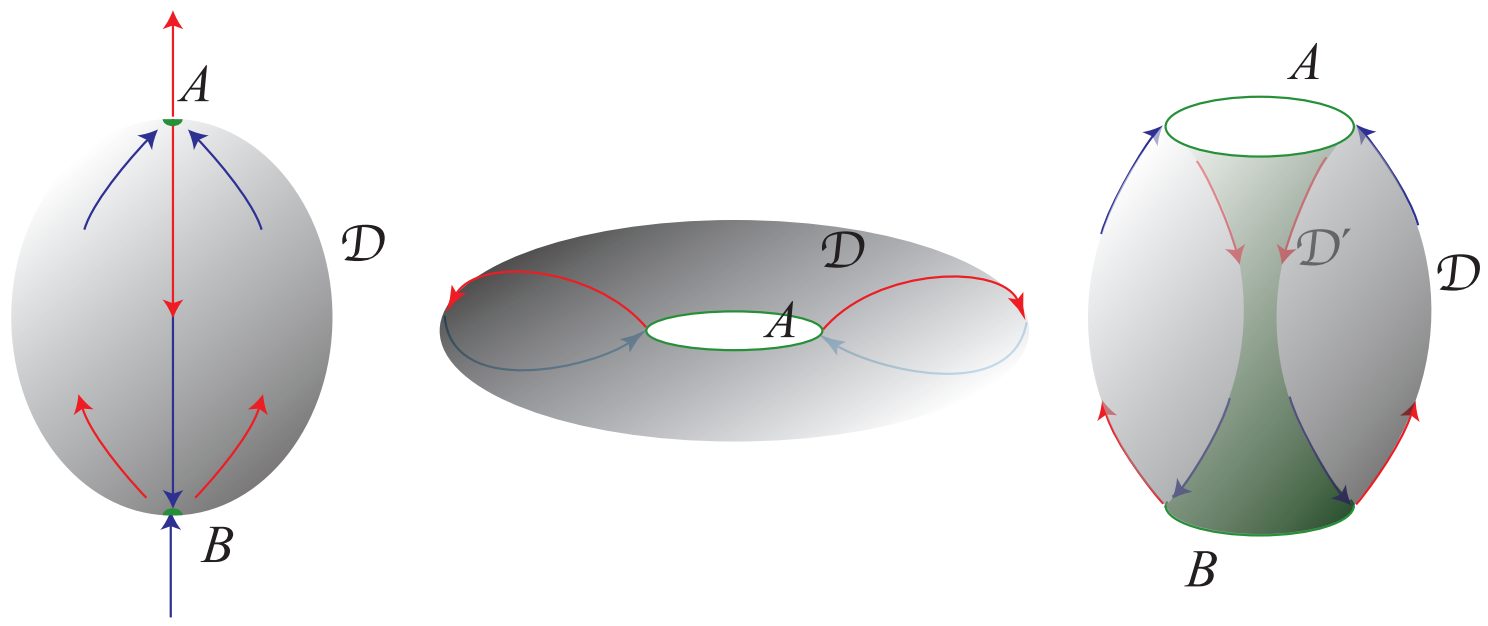

Figure 3: Resonance zones in the integrable approximation for a pair of fixed points, a single invariant circle or a pair of invariant circles.

When $A=B$ is a hyperbolic invariant circle and $W^{\mathrm{u}}(A) \cap W^{\mathrm{s}}(A) \neq \emptyset$, the resonance zone is a solid torus. This is also true when $A \neq B$, however, the boundary in this case is obtained from a pair of partial barriers, $\mathcal{D}$ constructed from $W^{\mathrm{s}}(A)$ and $W^{\mathrm{u}}(B)$ and $\mathcal{D}^{\prime}$ constructed from $W^{\mathrm{u}}(A)$ and $W^{\mathrm{s}}(B)$ (which we also assume intersect); as before the simple case where the partial barriers 
are saddle connections is sketched in Fig. 3. If $A$ is a fixed point and $B$ is an invariant circle, then $\mathcal{R}$ will typically be a ball, but a second fixed point or invariant circle will be needed to complete the resonance, and its boundary will consist of at least three partial barriers.

In the general case we require the following geometrical hypotheses.

(H1) $A$ and $B$ are normally hyperbolic invariant manifolds of dimension at most $\frac{n-1}{2}$;;

(H2) $W^{\mathrm{s}}(A)$ and $W^{\mathrm{u}}(B)$ are orientable, codimension-one submanifolds; and

(H3) the set of primary intersections $\mathcal{P}(A, B) \subset W^{\mathrm{s}}(A) \cap W^{\mathrm{u}}(B)$ is transverse.

To construct partial barrier $\mathcal{D}$, begin by selecting a pair of proper boundaries $\sigma$ and $\gamma$ such that the associated fundamental domains $\mathcal{S}=\mathcal{S}_{\sigma}(A)$ and $\mathcal{U}=\mathcal{U}_{\gamma}(B)$ are in "standard position", i.e., such that

(H4) $\kappa(\mathcal{S}, \mathcal{U})=0$ and the set $\eta \equiv \mathcal{S} \cap \mathcal{U} \subset \mathcal{P}$ a neat submanifold of $\mathcal{S}$ and $\mathcal{U}$ :

$$
\partial \eta \subset \partial \mathcal{S} \cap \partial \mathcal{U}
$$

This condition is sketched in Fig. 4 .

The simplest case corresponds to the primary intersection set containing a proper boundary, for then we can choose $\gamma=\sigma \subset \mathcal{P}$. In this case $(\mathbf{H} 4)$ is automatically satisfied since $\partial(\mathcal{S} \cap \mathcal{U})=\emptyset$. Recall that this is what is typically done for the two-dimensional case, as shown in Fig. 1. However $\mathcal{P}$ does not always contain a proper boundary, i.e., a curve with homology $(0,1)$; in particular, we commonly observe that $\mathcal{P}$ contains families of curves that spiral asymptotically from $B$ to $A$, as sketched in Fig. 2 [LM00, LM03]. This also must occur when the map is a Poincaré map of an autonomous flow, since heteroclinic points of the map lie on heteroclinic orbits of the flow [Mac94].

When resonance zone cannot have a boundary that consists solely of pieces of stable and unstable manifolds we must add a cap $\mathcal{C}$ to construct a partial barrier,

$$
\mathcal{D}=W_{\sigma}^{\mathrm{s}}(A) \cup W_{\gamma}^{\mathrm{u}}(B) \cup \mathcal{C}
$$

that will be one of the boundaries of $\mathcal{R}$, see Fig. 4. We assume that is it possible to choose the cap $\mathcal{C}$ so that

(H5) $\mathcal{C}$ is a codimension-one submanifold with boundary

$$
\partial \mathcal{C}=\gamma \cup \sigma
$$

(H6) $\mathcal{C} \cap W_{\sigma}^{\mathrm{s}}(A)=\sigma$ and $\mathcal{C} \cap \operatorname{cl}\left(W_{\gamma}^{\mathrm{u}}(B)\right)=\gamma$.

\footnotetext{
${ }^{4}$ A submanifold with boundary $V \subset W$ is neat if it is closed in $W$ and its boundary $\partial V$ is contained in the boundary $\partial W$ of $W$.
} 


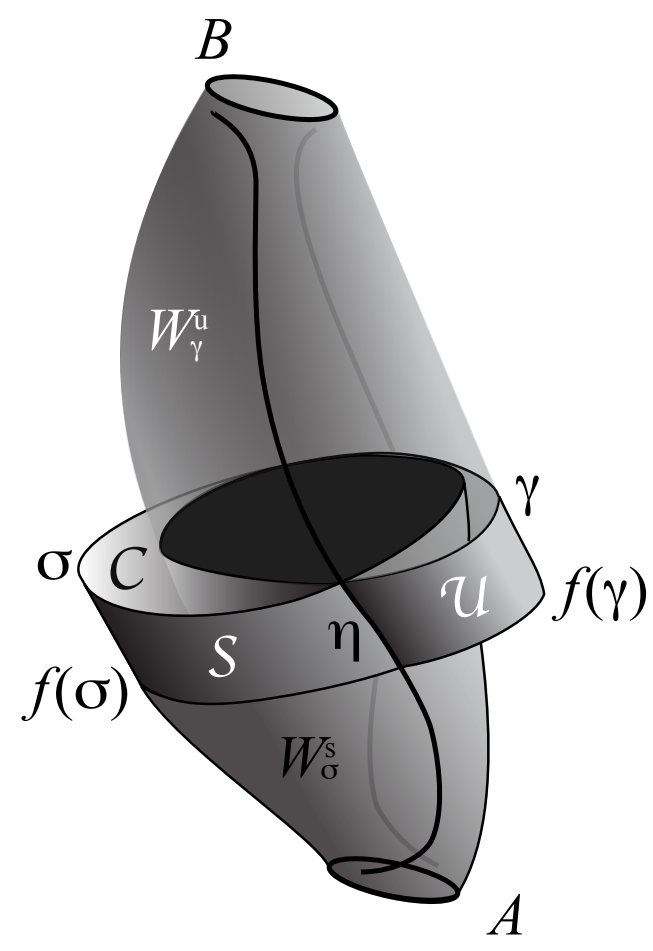

Figure 4: Partial barrier constructed from $W_{\sigma}^{\mathrm{s}}(A), W_{\gamma}^{\mathrm{u}}(B)$, and a cap $\mathcal{C}$.

For the three-dimensional case the partial barrier under these assumptions is topologically a sphere when $A$ and $B$ are fixed points, like that sketched in Fig. 3. When $A=B$ is an invariant circle then $\mathcal{D}$ is a torus, and when $A \neq B$ are invariant circles, $\mathcal{D}$ is an annulus bounded by the circles.

In the exceptional case that the set of primary intersections includes a proper boundary, we choose $\sigma=\gamma \subset \mathcal{P}$. In this case, $\mathcal{C}=\emptyset$, and the partial barrier is still given by (16).

\section{Lobe Volume}

If the map is exact area-preserving, the lobe volume depends only upon the orbit of the manifolds, and can be computed using the generator $S$ of (1) [MMP84, MMP87, Eas91. In this section we will show that the computation of lobe volume for the exact volume-preserving case will reduce to the integral of the one-form $\lambda$ of (2) along the primary intersection curves.

Before proceeding, in $\$ 5.2$, to obtain the formula for lobe volumes, we first derive an iterative formula relating the surface integral of $\alpha$ over a submanifold to those over its images. 


\subsection{Iterative Formula}

Here we obtain a fundamental iteration formula for the integral of $\alpha$ over a codimension-one submanifold $\mathcal{G}$ that is arbitrary, except that its boundary can be written as $\partial \mathcal{G}=\gamma \cup f(\gamma) \cup \eta$, i.e., as the union of two types of pieces: a manifold $\gamma$ and its image, and the remaining part $\eta$ that is not related by iteration. This situation is sketched in Fig. 5 .

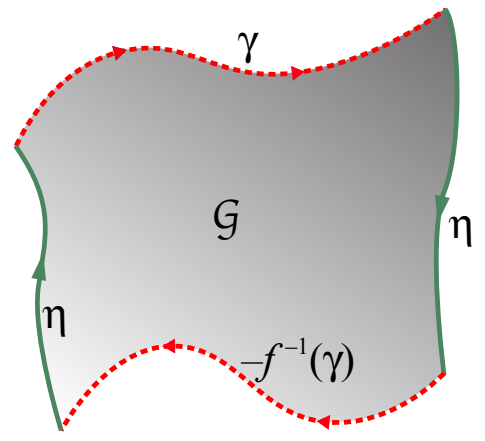

Figure 5: $\mathcal{G}$ is a submanifold such that a piece $\gamma$ of its boundary is related through $f$ with another piece with opposite orientation, $-f^{-1}(\gamma)$. In the diagram, an orientation of $\mathcal{G}$ induces an orientation on the boundary, and so $\partial \mathcal{G}=\gamma-f^{-1}(\gamma)+\eta$.

Lemma 3. Suppose $f: M \rightarrow M$ is an exact volume-preserving diffeomorphism, (2), and $\mathcal{G}$ is an oriented codimension-one submanifold with boundary $\partial \mathcal{G}=\gamma-f(\gamma)+\eta$, where $\gamma$ and $\eta$ are codimension-two submanifolds. Then, for any $t \in \mathbb{N}$

$$
\begin{aligned}
\int_{\mathcal{G}} \alpha+\int_{\gamma} \lambda & =-\sum_{k=0}^{t-1} \int_{\eta}\left(f^{k}\right)^{*} \lambda+\int_{f^{t}(\mathcal{G})} \alpha+\int_{f^{t}(\gamma)} \lambda \\
& =\sum_{k=-1}^{-t} \int_{\eta}\left(f^{k}\right)^{*} \lambda+\int_{f^{-t}(\mathcal{G})} \alpha+\int_{f^{-t}(\gamma)} \lambda .
\end{aligned}
$$

Proof. The composition formula (3) implies that

$$
\left(f^{t}\right)^{*} \alpha-\alpha=\sum_{k=0}^{t-1}\left(f^{k}\right)^{*} d \lambda .
$$

Integration of this relation over the region $\mathcal{G}$ and applying Stokes' theorem gives

$$
\int_{\mathcal{G}}\left(f^{t}\right)^{*} \alpha-\int_{\mathcal{G}} \alpha=\sum_{k=0}^{t-1} \int_{\eta+\gamma-f(\gamma)}\left(f^{k}\right)^{*} \lambda .
$$

The integrals over the images of the boundary curves $\gamma$ are a telescoping sum, so that

$$
\int_{\mathcal{G}}\left(f^{t}\right)^{*} \alpha-\int_{\mathcal{G}} \alpha=\sum_{k=0}^{t-1} \int_{\eta}\left(f^{k}\right)^{*} \lambda+\int_{\gamma} \lambda-\int_{\gamma}\left(f^{t}\right)^{*} \lambda .
$$


Upon noting that, for example,

$$
\int_{\mathcal{G}}\left(f^{t}\right)^{*} \alpha=\int_{f^{t}(\mathcal{G})} \alpha
$$

we see that the equation above is just the first line of 18 rearranged.

The remaining result can be obtained by a similar iteration, but backwards. Note that (2) implies $\alpha-\left(f^{-1}\right)^{*} \alpha=d\left(\left(f^{-1}\right)^{*} \lambda\right)$; this can be iterated to give

$$
\alpha-\left(f^{-t}\right)^{*} \alpha=\sum_{k=-1}^{-t}\left(f^{k}\right)^{*} d \lambda .
$$

Integrating this relation over $\mathcal{G}$, as before, and rearranging gives the final line of 18 .

\subsection{Lobe Volume Formula}

We continue to assume that $f$ obeys $(\mathbf{H 1})-(\mathbf{H 3})$ and has a partial barrier $\mathcal{D}$, (16), constructed from fundamental domains $\mathcal{S}$ and $\mathcal{U}$ and a cap $\mathcal{C}$ that obey $(\mathbf{H 4})-(\mathbf{H} 6)$. The turnstile for $\mathcal{D}$ is the union of the exit and incoming sets associated with the barrier; it is bounded by the fundamental domains, $\mathcal{S}$ and $\mathcal{U}$, and the cap $\mathcal{C}$ and its image $f(\mathcal{C})$

$$
\partial(\mathcal{E} \cup \mathcal{I})=\mathcal{U} \cup \mathcal{S} \cup \mathcal{C} \cup f(\mathcal{C})
$$

Though $\mathcal{C}$ is somewhat arbitrary, the lobe volume will be independent of this choice because its boundary contains both $\mathcal{C}$ and its image.

Since $W^{\mathrm{s}}(A)$ and $W^{\mathrm{u}}(B)$ have codimension one, in general they separate the manifold $M$. A consistent orientation for $W^{\mathrm{s}}(A)$ and $W^{\mathrm{u}}(B)$ will define an "outside" and an "inside" of the barrier $\mathcal{D}$. This in turn will induce an orientation of $\sigma$ and, by iteration, an orientation of $f(\sigma)$. In this case we can write $\partial \mathcal{S}=\sigma-f(\sigma)$ and $\partial \mathcal{U}=\gamma-f(\gamma)$.

The exit set is the portion of the turnstile where the unstable manifold is outside the stable manifold, we will use a + sign to denote this subset. The dividing set between exit and incoming lobes is the primary intersection $\eta=\mathcal{S}^{+} \cap \mathcal{S}^{-}=\mathcal{U}^{+} \cap \mathcal{U}^{-} \subset \mathcal{P}(A, B)$, which we have assumed is a submanifold obeying (15). Taking into account the orientation we write

$$
\mathcal{S}=\mathcal{S}^{+}+\mathcal{S}^{-} \text {and } \mathcal{U}=\mathcal{U}^{+}+\mathcal{U}^{-}
$$

see Fig. 6. Thus the exit lobe has boundary

$$
\partial \mathcal{E}=\mathcal{U}^{+}-\mathcal{S}^{+}+\mathcal{C}^{+}-f\left(\mathcal{C}^{+}\right)
$$

The primary intersection $\eta$ also divides the loops $\sigma$ and $\gamma$ into pieces that can be labeled \pm

$$
\sigma=\sigma^{+}+\sigma^{-} \text {and } \gamma=\gamma^{+}+\gamma^{-}
$$


as sketched in Fig. 6. Consequently, the fundamental domains and the cap have boundaries

$$
\begin{aligned}
\partial \mathcal{S}^{ \pm} & = \pm \eta+\sigma^{ \pm}-f\left(\sigma^{ \pm}\right), \\
\partial \mathcal{U}^{ \pm} & = \pm \eta+\gamma^{ \pm}-f\left(\gamma^{ \pm}\right), \\
\partial \mathcal{C}^{ \pm} & =\sigma^{ \pm}-\gamma^{ \pm} .
\end{aligned}
$$

We will compute the volume of the exit lobe 19 , but as we discussed in 44.1 , the volume of the incoming lobe is the same.
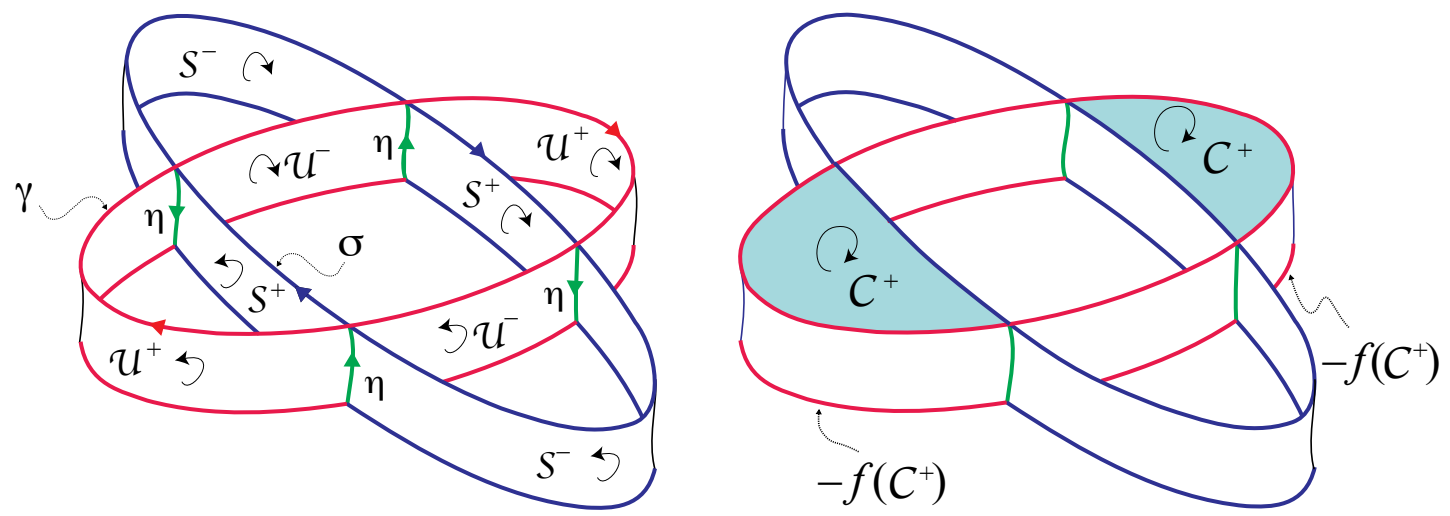

Figure 6: Three-dimensional lobes. On the left is illustrated the boundary of the exit and incoming lobes formed from a pair of fundamental domains that are subsets of stable and unstable manifolds. On the right a pair of caps are added to complete the exit lobe.

Theorem 4 (Lobe Volume). Suppose that $\mathcal{D}$ is a partial barrier for an exact volume-preserving map $f$, obeying (H1)-(H6) and such that the one-form $\lambda$ is regular on $W^{\mathrm{s}}(A)$ and $W^{\mathrm{u}}(B)$. Then the volume of the exit lobe $\mathcal{E}$ with the boundary (19) is

$$
\operatorname{Vol}(\mathcal{E})=\sum_{k \in \mathbb{Z}} \int_{\eta}\left(f^{k}\right)^{*} \lambda
$$

Proof. If $\mathcal{S}^{+}$and $\mathcal{U}^{+}$are pieces of fundamental domains with boundaries obeying $(20)$, then Stokes' theorem with $(2),(19)$, and $\partial \mathcal{C}^{+}=\sigma^{+}-\gamma^{+}$gives

$$
\begin{aligned}
\int_{\mathcal{E}} \Omega=\int_{\partial \mathcal{E}} \alpha & =\int_{\mathcal{U}^{+}} \alpha-\int_{\mathcal{S}^{+}} \alpha+\int_{\mathcal{C}^{+}} \alpha-\int_{f\left(\mathcal{C}^{+}\right)} \alpha \\
& =\int_{\mathcal{U}^{+}} \alpha-\int_{\mathcal{S}^{+}} \alpha-\int_{\mathcal{C}^{+}} \lambda \\
& =\int_{\mathcal{U}^{+}} \alpha+\int_{\gamma^{+}} \lambda-\int_{\mathcal{S}^{+}} \alpha-\int_{\sigma^{+}} \lambda .
\end{aligned}
$$


By (20), Lem. 3 applies to the terms in the last line of $(22)$, so that for all $t \in \mathbb{N}$,

$$
\begin{gathered}
\int_{\mathcal{U}^{+}} \alpha+\int_{\gamma^{+}} \lambda=\sum_{k=-1}^{-t} \int_{\eta}\left(f^{k}\right)^{*} \lambda+\int_{f^{-t}\left(\mathcal{U}^{+}\right)} \alpha+\int_{f^{-t}\left(\gamma^{+}\right)} \lambda, \\
\int_{\mathcal{S}^{+}} \alpha+\int_{\sigma^{+}} \lambda=-\sum_{k=0}^{t-1} \int_{\eta}\left(f^{k}\right)^{*} \lambda+\int_{f^{t}\left(\mathcal{S}^{+}\right)} \alpha+\int_{f^{t}\left(\sigma^{+}\right)} \lambda .
\end{gathered}
$$

Here we have selected the direction of iteration to take advantage of the contraction of the manifolds. Since by $(\mathbf{H 1}), \operatorname{dim}(A)$ and $\operatorname{dim}(B)$ are at most $\frac{n-1}{2}$, Lem. 2 implies that the $(n-1)$-form $\alpha$ is regular on $W^{\mathrm{s}}(A)$ and $W^{\mathrm{u}}(B)$. Thus, since $\operatorname{cl}\left(\mathcal{U}^{+}\right) \subset W^{\mathrm{u}}(B)$ and $\operatorname{cl}\left(\mathcal{S}^{+}\right) \subset W^{\mathrm{s}}(A)$ are compact Lem. 1 implies

$$
\lim _{t \rightarrow \infty} \int_{f^{-t}\left(\mathcal{U}^{+}\right)} \alpha=0, \quad \lim _{t \rightarrow \infty} \int_{f^{t}\left(\mathcal{S}^{+}\right)} \alpha=0 .
$$

Finally, since $\lambda$ is assumed to be regular on $W^{\mathrm{s}}(A)$ and $W^{\mathrm{s}}(B)$, then

$$
\lim _{t \rightarrow \infty} \int_{f^{-t}\left(\gamma^{+}\right)} \lambda=0, \quad \lim _{t \rightarrow \infty} \int_{f^{t}\left(\sigma^{+}\right)} \lambda=0 .
$$

After taking the limit in $(23)$ and substituting back into $(22)$, we find

$$
\int_{\mathcal{E}} \Omega=\sum_{k=-1}^{-\infty} \int_{\eta}\left(f^{k}\right)^{*} \lambda+\sum_{k=0}^{\infty} \int_{\eta}\left(f^{k}\right)^{*} \lambda,
$$

which is equivalent to (21). This concludes the proof.

Remark (1). The primary intersection between $A$ and $B$ is given by

$$
\mathcal{P}(A, B)=\bigcup_{k \in \mathbb{Z}} f^{k}(\eta)
$$

Therefore, under the assumptions of Thm. 4, (21) can be written

$$
\int_{\mathcal{E}} \Omega=\sum_{k \in \mathbb{Z}} \int_{\eta}\left(f^{k}\right)^{*} \lambda=\int_{\mathcal{P}(A, B)} \lambda .
$$

Remark (2). By Lem. 2, the $(n-2)$-form $\lambda$ is regular on the $(n-1)$-dimensional manifolds $W^{\mathrm{s}}(A)$ and $W^{\mathrm{s}}(B)$ whenever $\operatorname{dim}(A), \operatorname{dim}(B) \leq \frac{n}{2}-1$. This includes the case that $A$ and $B$ are fixed points and $n \geq 3$; however, $\lambda$ is not necessarily regular for $n=3$ when $A$ or $B$ are invariant circles. When $A=B$, the hypothesis that $\lambda$ is regular is probably not needed. It is only the difference

$$
\int_{f^{-t}\left(\gamma^{+}\right)} \lambda-\int_{f^{t}\left(\sigma^{+}\right)} \lambda
$$

that must limit to zero in 23 to obtain (21). This difference converges exponentially to zero if $f^{-t}\left(\gamma^{+}\right) \rightarrow A$ and $f^{t}\left(\sigma^{+}\right) \rightarrow A$ as $t \rightarrow \infty$ so that the domains of integration become identical. This 
is what happens for $n=2$ where $\lambda$ is the zero-form $S$, (1), MMP84, MMP87, and for the examples in $\$ 7$. It would be nice to show that it is always possible to select proper boundaries so that this is the case. When $A \neq B$, it is not obvious whether the requirement that $\lambda$ be regular can always be satisfied. Since any closed form can be added to $\lambda$ without changing (2), it may be possible to use this freedom to make $\lambda$ regular. One way to do this would be to have $\left.\lambda\right|_{A}=\left.\lambda\right|_{B}=0$.

Thm. 4 implies that the volume of an $n$-dimensional exit set can be computed by integrating along the $(n-2)$-dimensional primary intersection set. In general, the volume of an $n$-dimensional lobe can be computed by integrating the $(n-2)$-form $\lambda$ on the primary intersection $\mathcal{P}(A, B)$ that is an immersed submanifold of dimension $n-2$. Moreover, the flux across a partial barrier determined by the manifolds $W^{\mathrm{s}}(A)$ and $W^{\mathrm{u}}(B)$ is independent of the selection of the fundamental domains and of the cap $\mathcal{C}$.

Finally, note that Thm. 4 also applies to the case that the cap $\mathcal{C}$ is the empty set. This would be the case if $\mathcal{P}$ includes a proper loop, for then we can select $\sigma=\gamma \in \mathcal{P}$ and $\mathcal{D}=W_{\sigma}^{\mathrm{s}}(A) \cup W_{\sigma}^{\mathrm{u}}(B)$.

\section{Melnikov Flux}

In this section, we show how our formula (21) for the lobe volume limits to well-known Melnikov results for maps with a near saddle-connection. This is well-known for the two-dimensional case [MM88] and is implicit in the theory developed in [LM00] for the three-dimensional case. To prepare for the result, we recall some notation and results of [LM03, LMRR08].

Suppose $f_{\delta}: M \rightarrow M$ is a smooth family of exact volume-preserving diffeomorphisms (2) satisfying (H1)-(H3) of 4.4 for each $0<|\delta|<\delta_{0}$. In addition, suppose that the map $f_{0}$ has a saddle connection

$$
\Sigma \subset W^{\mathrm{s}}\left(A_{0}\right) \cap W^{\mathrm{u}}\left(B_{0}\right) .
$$

between the normally hyperbolic invariant manifolds $A_{0}$ and $B_{0}$ like those sketched in Fig. 3. As usual, we assume that $\operatorname{dim}(\Sigma)=n-1$ and $\operatorname{dim}\left(A_{0}\right), \operatorname{dim}\left(B_{0}\right) \leq \frac{n-1}{2}$.

By (H3), when $\delta>0$ the saddle connection splits into the stable and unstable manifolds $W^{\mathrm{s}}\left(A_{\delta}\right)=W^{\mathrm{s}}\left(A_{\delta}, f_{\delta}\right)$ and $W^{\mathrm{u}}\left(B_{\delta}\right)=W^{\mathrm{u}}\left(B_{\delta}, f_{\delta}\right)$ of the perturbed invariant sets. The splitting of these manifolds is computed, to lowest order, by the classical Melnikov function.

Specifically, suppose there is a fundamental domain $\mathcal{F} \subset \Sigma$ and two diffeomorphisms $\psi_{\delta}^{s, u}: \mathcal{F} \subset$ $\Sigma \rightarrow M$ (adapted deformations), such that

$$
\mathcal{U}_{\delta}=\psi_{\delta}^{\mathrm{u}}(\mathcal{F}) \text { and } \mathcal{S}_{\delta}=\psi_{\delta}^{\mathrm{s}}(\mathcal{F})
$$

are fundamental domains of the perturbed manifolds $W^{\mathrm{s}}\left(A_{\delta}\right)$ and $W^{\mathrm{u}}\left(B_{\delta}\right)$. When $\delta=0$, we can take $\psi_{0}^{\mathrm{u}, \mathrm{s}}$ to be the trivial inclusion $\psi_{\delta}^{s, u}: \mathcal{F} \subset \Sigma \rightarrow M, \psi_{0}^{\mathrm{u}, \mathrm{s}}(\xi)=\xi$. so that

$$
\mathcal{U}_{0}=\mathcal{S}_{0}=\mathcal{F}
$$


We notice that the intersection index $\kappa\left(\mathcal{S}_{0}, \mathcal{U}_{0}\right)=\kappa(\mathcal{F}, \mathcal{F})=0$, since $\mathcal{F}$ is a fundamental domain, but this need not be true for $\mathcal{S}_{\delta}$ and $\mathcal{U}_{\delta}$, unless the deformations were chosen carefully. Nevertheless, as in (H3), we assume that the set of primary intersection $\eta_{\delta}$ is transverse for small $\delta$. As we will see, the limiting set $\eta_{0} \cap \mathcal{F}$ is the zero set of a Melnikov function.

To first order in $\delta$ the only relevant quantities associated with $f_{\delta}$ and the deformations $\psi_{\delta}^{\mathrm{s}, \mathrm{u}}$ are their vector fields with respect to $\delta$. The perturbation of the map $f_{0}$ away from $\delta=0$ is measured by its perturbation vector field

$$
\left.X(x) \equiv \frac{\partial}{\partial \delta}\right|_{\delta=0} f_{\delta}\left(f_{0}^{-1}(x)\right)
$$

for each $x \in M$. The perturbations of the deformations $\psi_{\delta}^{\mathrm{s}, \mathrm{u}}$ are similarly defined by the vector fields

$$
Z^{\mathrm{s}, \mathrm{u}}(\xi)=\left.\frac{\partial}{\partial \delta}\right|_{\delta=0} \psi_{\delta}^{\mathrm{s}, \mathrm{u}}(\xi)
$$

for each $\xi \in \mathcal{F}$, using the assumption that $\psi_{0}^{\mathrm{s}, \mathrm{u}}=i d$ on $\mathcal{F}$.

Up to vectors tangent to the saddle connection, these vector fields are related by the iterative formulas LMRR08]

$$
\begin{aligned}
& \left(f_{0}\right)_{*} Z^{\mathrm{u}, \mathrm{s}}-Z^{\mathrm{u}, \mathrm{s}}+X_{0} \in T \mathcal{F}, \\
& Z^{\mathrm{u}, \mathrm{s}}-f_{0}^{*} Z^{\mathrm{u}, \mathrm{s}}+f_{0}^{*} X_{0} \in T \mathcal{F} .
\end{aligned}
$$

To see this, note that since $\Sigma$ is invariant under $f_{0}$, the functions $\tilde{\psi}_{\delta}^{\mathrm{s}, \mathrm{u}}=f_{\delta} \circ \psi_{\delta}^{\mathrm{s}, \mathrm{u}} \circ f_{0}^{-1}$ are also adapted deformations, mapping $\Sigma$ to $W_{\delta}^{\mathrm{s}, \mathrm{u}}$. In either case, the function $C_{\delta}=\tilde{\psi}_{\delta}^{-1} \circ \psi_{\delta}$ defines a curve in $\mathcal{F}$, so that the vector $\frac{d}{d \delta} C_{\delta}$ is in $T \mathcal{F}$. Computing this derivative implies that

$$
\left[\frac{\partial}{\partial \delta} \tilde{\psi}_{\delta}(x)-\frac{\partial}{\partial \delta} \psi_{\delta}(x)\right]_{\delta=0} \in T_{\xi} \mathcal{F}
$$

However,

$$
\left[\frac{\partial}{\partial \delta} \tilde{\psi}_{\delta}(x)\right]_{\delta=0}=X_{0}(\xi)+D f_{0}\left(f_{0}^{-1}(\xi)\right) Z\left(f_{0}^{-1}(\xi)\right)
$$

Recalling the definition of the pullback and pushfoward (see 61) and combining these two results gives the pair of formulas 26 and (27).

Since the maps $\psi^{\mathrm{s}, \mathrm{u}}$ describe the deformations of the stable and unstable manifolds, the "velocity" of the splitting with $\delta$ is given by the deformation vector field

$$
\Delta(\xi)=Z^{\mathrm{u}}(\xi)-Z^{\mathrm{s}}(\xi)
$$

The stable and unstable manifolds are thus "flowing" with respect to $\delta$ according to the vector fields $Z^{\mathrm{s}, \mathrm{u}}$ and they split at a rate $\Delta$. This flow induces a "flux" with respect to the volume form $\Omega$ defined by

$$
\Phi \equiv i_{\Delta} \Omega .
$$


The form $\Phi$ is the flux form of the deformation $\Delta$.

Though the expression for the splitting velocity $\Delta$ depends on the choice of adapted deformations, the form $i_{\Delta} \Omega$ is well-defined on $\Sigma$ and is independent of the choice of $\psi^{s, u}$ [LM03]. In addition, from the definition (28) with (26) we have

$$
f_{0}^{*} \Delta-\Delta \in T \mathcal{F}
$$

which implies that

$$
f_{0}^{*}\left(i_{\Delta} \Omega\right)=i_{f_{0}^{*} \Delta}\left(f_{0}^{*} \Omega\right)=i_{f_{0}^{*} \Delta} \Omega=i_{\Delta} \Omega \text { on } \Sigma,
$$

i.e., the flux is invariant under $f_{0}$.

The flux is our primary measure of the splitting; indeed as we will show below, the integral of $\Phi$ is related to the lobe volume. Since $f_{\delta}$ is exact, the net flux across a fundamental domain vanishes.

Proposition 5 ([LM03]). If $\mathcal{F}$ is a fundamental domain in $\Sigma$ then

$$
\int_{\mathcal{F}} \Phi=0
$$

The classical Melnikov function is defined relative to a choice of an adapted normal vector field $\mathbf{n}: \Sigma \rightarrow T M$ [LM00]. Recall that vector field is adapted to $f_{0}$ if, for all vector fields $Y$ and all points $\xi \in \Sigma$,

$$
f_{0}^{*}\langle\mathbf{n}, Y\rangle=\left\langle\mathbf{n}, f_{0}^{*} Y\right\rangle .
$$

Given an inner product $\langle$,$\rangle and such a vector field, the Melnikov function M_{\mathbf{n}}: \Sigma \rightarrow \mathbb{R}$ is simply the normal component of the deformation,

$$
M_{\mathbf{n}}=\langle\mathbf{n}, \Delta\rangle .
$$

Since $\mathbf{n}$ is a vector field in the algebraic normal bundle of $\Sigma, M_{\mathbf{n}}$ is a measure of the speed of splitting in the normal direction. Conditions (30) and (31) together imply that $M_{\mathbf{n}}$ is invariant under $f_{0}$, that is, $M_{\mathbf{n}} \circ f_{0}=M_{\mathbf{n}}$. One common choice for $\mathbf{n}$ is the gradient of an invariant of $f_{0}$ (if one exists) [LM00, but any adapted normal vector field gives a Melnikov function with the same zero set.

The main point of Melnikov theory is that if a point $\xi \in \Sigma$ is a nondegenerate zero of $M_{\mathbf{n}}$, the stable and unstable manifolds $W^{\mathrm{u}}\left(B_{\delta}\right)$ and $W^{\mathrm{s}}\left(A_{\delta}\right)$ intersect transversally near $\xi$ when $\delta$ is small enough. The flux form is simply related to the classical Melnikov formula by

$$
\Phi=M_{\mathbf{n}} \omega_{\mathbf{n}}
$$

where $\omega_{\mathbf{n}}$ is the natural $(n-1)$-volume form on $\Sigma$ induced by $\mathbf{n}$ [LM03, LMRR08,

$$
\omega_{\mathbf{n}}=\frac{1}{\langle\mathbf{n}, \mathbf{n}\rangle} i_{\mathbf{n}} \Omega
$$


When $\mathbf{n}$ is an adapted normal, $\omega_{\mathbf{n}}$ is invariant under $f_{0}: f_{0}^{*} \omega_{\mathbf{n}}=\omega_{\mathbf{n}}$ on $\Sigma$.

The iterative formula (26) implies [LM03, LMRR08]

$$
\Delta-\sum_{k=-\infty}^{\infty}\left(f_{0}^{*}\right)^{k} X \in T \mathcal{F} .
$$

Indeed, the function $\Delta$ can be thought of as a section on a normal bundle. It was proven in LMRR08] that if one considers both $\Delta$ and the series $\sum_{k=-\infty}^{\infty}\left(f_{0}^{*}\right)^{k} X$ as sections, then they are the same function.

That is, the deformation vector field is, up to tangent vectors, given by the infinite sum above. This infinite sum is geometrically convergent since the invariant sets $A_{0}$ and $B_{0}$ are normally hyperbolic. By (32) and (35) the Melnikov function can be written

$$
M_{\mathbf{n}}=\sum_{k=-\infty}^{\infty}\left\langle\mathbf{n},\left(f_{0}^{*}\right)^{k} X\right\rangle=\sum_{k=-\infty}^{\infty}\langle\mathbf{n}, X\rangle \circ f_{0}^{k}
$$

since, by definition, $\langle\mathbf{n}, Y\rangle=0$ for any $Y \in T \mathcal{F}$.

There is considerable freedom in the selection of the adapted deformations $\psi_{\delta}^{\mathrm{s}, \mathrm{u}}$; we may choose any functions from $\mathcal{F}$ to $W^{\mathrm{s}, \mathrm{u}}$ that reduce to the identity when $\delta=0$. Given an given adapted normal $\mathbf{n}$, there is a natural choice: for each $\xi \in \mathcal{F}$ choose $\tilde{\psi}_{\delta}^{\mathrm{s}}(\xi)\left(\tilde{\psi}_{\delta}^{\mathrm{u}}(\xi)\right)$ to belong to the intersection of the stable (unstable) manifold with the line generated by $\mathbf{n}(\xi)$. Under this choice $\widetilde{\Delta}=\phi \mathbf{n}$ for some function $\phi: \Sigma \rightarrow \mathbb{R}$ to be determined.

Since $\tilde{\Delta}$ is also a deformation vector field, 35 implies that $\Delta-\widetilde{\Delta} \in T \mathcal{F}$, so that $M_{\mathbf{n}}=\langle\mathbf{n}, \tilde{\Delta}\rangle$, as well. From this, we conclude that $M_{\mathbf{n}}=\langle\mathbf{n}, \mathbf{n}\rangle \phi$ and therefore $\widetilde{\Delta}$ has to be of the form

$$
\widetilde{\Delta}=\left(\frac{M_{\mathbf{n}}}{\langle\mathbf{n}, \mathbf{n}\rangle}\right) \mathbf{n} .
$$

We are now prepared to state relate these old results to Thm. 4 showing that an integral of the flux form $\Phi$ gives the rate of growth of the lobe volume with $\delta$.

Theorem 6. Let $f_{\delta}$ be a $C^{1}$ family of exact volume-preserving diffeomorphisms with partial barriers $\mathcal{D}_{\delta}$ and exit sets $\mathcal{E}_{\delta}$ obeying $(\boldsymbol{H} \mathbf{1})-(\boldsymbol{H} \boldsymbol{6})$, such that $f_{0}$ has a saddle connection

$$
\Sigma \subset W^{\mathrm{s}}\left(A_{0}\right) \cap W^{\mathrm{u}}\left(B_{0}\right)
$$

Lett $M_{\mathbf{n}}$ be a Melnikov function (32) for $f_{\delta}$ and $\mathcal{F}^{+}=\mathcal{F} \cap M_{\mathbf{n}}^{-1}\left(\mathbb{R}^{+}\right)$be the set of points in the fundamental domain $\mathcal{F}$ for which the Melnikov function is positive. Then

$$
\left.\frac{d}{d \delta}\right|_{\delta=0} \int_{\mathcal{E}_{\delta}} \Omega=\int_{\mathcal{F}^{+}} \Phi=\frac{1}{2} \int_{\mathcal{F}}\left|M_{\mathbf{n}}\right| \omega_{\mathbf{n}} .
$$


Proof. By assumption $f_{\delta}$ is exact-volume preserving so there exists a family of $(n-2)$-forms $\lambda_{\delta}$ such that

$$
f_{\delta}^{*} \alpha-\alpha=d \lambda_{\delta}
$$

Note that since $f_{\delta}$ is assumed to be smooth with respect to $\delta$, we can take $\lambda_{\delta}$ to be smooth as well.

Let $\partial \mathcal{F}=\sigma_{0}-f_{0}\left(\sigma_{0}\right)$ for the proper boundary $\sigma_{0}$ and define $\sigma_{0}^{+}=\sigma_{0} \cap \mathcal{F}^{+}$so that the boundary of $\mathcal{F}^{+}$is of the form

$$
\partial \mathcal{F}^{+}=\sigma_{0}^{+}-f_{0}\left(\sigma_{0}^{+}\right)+\eta_{0}
$$

According to 22

$$
\int_{\mathcal{E}_{\delta}} \Omega=\int_{\mathcal{U}_{\delta}^{+}} \alpha-\int_{\mathcal{S}_{\delta}^{+}} \alpha+\int_{\gamma_{\delta}^{+}} \lambda_{\delta}-\int_{\sigma_{\delta}^{+}} \lambda_{\delta}
$$

Using the adapted deformations $\psi_{\delta}^{\mathrm{s}, \mathrm{u}}$ to map $\mathcal{F}^{+}$to $\mathcal{S}_{\delta}^{+}$and $\mathcal{U}_{\delta}^{+}$, respectively, then gives

$$
\int_{\mathcal{E}_{\delta}} \Omega=\int_{\mathcal{F}^{+}}\left[\left(\psi_{\delta}^{\mathrm{u}}\right)^{*} \alpha-\left(\psi_{\delta}^{\mathrm{s}}\right)^{*} \alpha\right]+\int_{\sigma_{0}^{+}}\left[\left(\psi_{\delta}^{\mathrm{u}}\right)^{*} \lambda_{\delta}-\left(\psi_{\delta}^{\mathrm{s}}\right)^{*} \lambda_{\delta}\right] .
$$

Differentiate this relation with respect to $\delta$ and use the definition (28) to obtain

$$
\left.\frac{\partial}{\partial \delta}\right|_{\delta=0} \int_{\mathcal{E}_{\delta}} \Omega=\int_{\mathcal{F}^{+}} L_{\Delta} \alpha+\int_{\sigma_{0}^{+}} L_{\Delta} \lambda_{0}
$$

where $L_{\Delta}$ is the Lie derivative 63.

Cartan's formula (64) implies that

$$
\begin{aligned}
L_{\Delta} \alpha & =i_{\Delta} d \alpha+d\left(i_{\Delta} \alpha\right)=i_{\Delta} \Omega+d\left(i_{\Delta} \alpha\right), \\
L_{\Delta} \lambda_{0} & =i_{\Delta} d \lambda_{0}+d\left(i_{\Delta} \lambda_{0}\right)=i_{\Delta}\left(f_{0}^{*} \alpha\right)-i_{\Delta} \alpha+d\left(i_{\Delta} \lambda_{0}\right),
\end{aligned}
$$

where we used $d \alpha=\Omega$ and the exactness of $f_{0}$. Substitution of these results into 42 yields

$$
\left.\frac{\partial}{\partial \delta}\right|_{\delta=0} \int_{\mathcal{E}_{\delta}} \Omega=\int_{\mathcal{F}^{+}} i_{\Delta} \Omega+N
$$

where

$$
N \equiv \int_{\partial \mathcal{F}^{+}} i_{\Delta} \alpha+\int_{\sigma_{0}^{+}} i_{\Delta}\left(f_{0}^{*} \alpha\right)-\int_{\sigma_{0}^{+}} i_{\Delta} \alpha+\int_{\partial \sigma_{0}^{+}} i_{\Delta} \lambda_{0} .
$$

Consequently, in order to prove the theorem we must finally show that $N=0$. Using $(39)$ for $\partial \mathcal{F}^{+}$, then (43) becomes

$$
\begin{aligned}
N & =\int_{\sigma_{0}^{+}}\left[i_{\Delta}\left(f_{0}^{*} \alpha\right)-f_{0}^{*}\left(i_{\Delta} \alpha\right)\right]+\int_{\eta_{0}} i_{\Delta} \alpha+\int_{\partial \sigma_{0}^{+}} i_{\Delta} \lambda_{0} \\
& =\int_{\sigma_{0}^{+}} i_{\left(\Delta-f_{0}^{*} \Delta\right)}\left(f_{0}^{*} \alpha\right)+\int_{\eta_{0}} i_{\Delta} \alpha+\int_{\partial \sigma_{0}^{+}} i_{\Delta} \lambda_{0} .
\end{aligned}
$$


Note that this expression for $N$ is independent of the choice of $\alpha$. In order words, if $\widetilde{\alpha}$ is any form such that $d \widetilde{\alpha}=\Omega$ and $f_{\delta}^{*} \widetilde{\alpha}-\widetilde{\alpha}=d \widetilde{\lambda}_{\delta}$ then we may begin again using the new forms in the computation of (40), obtaining finally (44) with $\alpha \rightarrow \widetilde{\alpha}$ and $\lambda_{0} \rightarrow \tilde{\lambda}_{0}$. In particular, if $f_{0}$ is exact with respect to $\alpha$, then it is also exact with respect to $\widetilde{\alpha}=\left(f_{0}^{*}\right)^{k-1} \alpha$, for all $k \in \mathbb{N}$. Using this new form in (44) gives

$$
N=\int_{\sigma_{0}^{+}} i_{\left(\Delta-f_{0}^{*} \Delta\right)}\left(f_{0}^{*}\right)^{k} \alpha+\int_{\eta_{0}} i_{\Delta}\left(f_{0}^{*}\right)^{k} \alpha+\int_{\partial \sigma_{0}^{+}} i_{\Delta}\left(f_{0}^{*}\right)^{k} \lambda_{0}
$$

Finally, note that $N$ is also independent of the choice of $\Delta$ : since 41) is independent of the choice of adapted deformations, (42) is also independent of the choice of $\Delta$-this equation and the subsequent ones are valid for any deformation $\tilde{\Delta}$ that comes from a pair of adapted deformations $\tilde{\psi}_{\delta}^{\mathrm{s}, \mathrm{u}}$. In particular, we will use the deformation (37), so that $\Delta \rightarrow \tilde{\Delta}$, which is normal to $\mathcal{F}$ and thus $\left.\tilde{\Delta}\right|_{\eta_{0}}=\tilde{\Delta}_{\sigma_{0}^{+}}=0$ since $\partial \sigma_{0}^{+} \subset \eta_{0} \subset \mathcal{F}$. Thus if we define $Y=\tilde{\Delta}-f_{0}^{*} \tilde{\Delta}$ then $Y \in T \mathcal{F}$ by 30 and

$$
N=\int_{\sigma_{0}^{+}} i_{Y}\left(f_{0}^{*}\right)^{k} \alpha
$$

Since the $(n-1)$-form $\alpha$ is regular when $\operatorname{dim}\left(A_{0}\right), \operatorname{dim}\left(B_{0}\right) \leq \frac{n-1}{2}$, as assumed in (H1), Lem. 1 implies that as $k \rightarrow \infty, N \rightarrow 0$; however, $N$ is independent of $k$, therefore $N \equiv 0$.

\section{$7 \quad$ Examples}

Here we give two simple examples demonstrating that 21) gives the expected results for the lobe volume. In the first case, the lobes are simply given by the cross product of the lobes for an area preserving map with a circle. In the second, the map is the time $T$ map of a nonautonomous flow.

\subsection{Semidirect Product with a Twist Map}

An area-preserving, twist map $(X, Y)=g(x, y)$ can be obtained from an implicit generating function $G(x, X)$ through the equations

$$
Y=\partial_{2} G(x, X), \quad y=-\partial_{1} G(x, X)
$$

provided that $G$ satisfies the twist condition $\partial_{1} \partial_{2} G \neq 0$. A map generated in this way is also exact in the sense of (1). Indeed we may choose $\nu=y d x$ and the zero-form

$$
S(x, y)=G(x, X(x, y))
$$

for in this case

$$
g^{*} \nu-\nu=Y d X-y d x,
$$


and

$$
d S(x, y)=\partial_{1} G(x, X) d x+\partial_{2} G(x, X) d X,
$$

which are equivalent to (45).

The map $g$ can be extended to an exact volume-preserving map on $M=\mathbb{R}^{2} \times \mathbb{S}$ by introducing an angle $\theta \in \mathbb{S} \equiv \mathbb{R} /(2 \pi \mathbb{Z})$, and some appropriate dynamics. A simple case is the semidirect product

$$
(X, Y, \Theta)=f(x, y, \theta)=(g(x, y), \theta+\rho(x, y)),
$$

where $\rho$ is the local rotation number. Now $f$ is an exact volume-preserving map with the volume form $\Omega=d x \wedge d y \wedge d \theta$. For example, using the two form

$$
\alpha=d\left(S \circ f^{-1}\right) \wedge d \theta-y d x \wedge d \theta
$$

so that $\Omega=d \alpha$, and $d S=Y d X-y d x$ gives

$$
\begin{aligned}
f^{*} \alpha-\alpha & =d S \wedge d \Theta-Y d X \wedge d \Theta-d\left(S \circ f^{-1}\right) \wedge d \theta+y d x \wedge d \theta \\
& =-d\left(S \circ f^{-1}\right) \wedge d \theta-y\left(\partial_{y} \rho\right) d x \wedge d y .
\end{aligned}
$$

Therefore, $f$ obeys 2 with the one form

$$
\lambda=-\left(S \circ f^{-1}\right) d \theta-\phi(x, y) y d y .
$$

where $\phi$ is a function such that $\partial_{x} \phi=\partial_{y} \rho$.

Because of the semidirect product structure of (47), if $a$ is a saddle fixed point of $g$, then $A=a \times \mathbb{S}$ is a normally hyperbolic invariant circle for $f$. Similarly if $\mathcal{R}$ is a resonance zone for $g$ (recall for example Fig. 1) then $\overline{\mathcal{R}}=\mathcal{R} \times \mathbb{S}$ is a resonance zone for $f$. Moreover, if $\mathcal{E}$ is the exit set for $\mathcal{R}$ that is delineated by a pair of primary intersection points $p$ and $q$, then $\overline{\mathcal{E}}=\mathcal{E} \times \mathbb{S}$ is the exit set for the three-dimensional resonance zone of $f$. Moreover, since for each $(x, y)$ the map $\Theta=\theta+\rho$ is a homeomorphism of the circle $\mathbb{S}$, the primary intersection manifold of the fundamental domains for $f$ is the submanifold

$$
\eta=\eta_{p}-\eta_{q}
$$

where $\eta_{p}=\{p\} \times \mathbb{S}$ and $\eta_{q}=\{q\} \times \mathbb{S}$, and $p, q$ are in the primary homoclinic orbits for $g$, recall Fig. 1 .

Thus we can apply Thm. 4 to compute the volume of $\mathcal{E} \times \mathbb{S}$

$$
\operatorname{Vol}(\overline{\mathcal{E}})=\sum_{k \in \mathbb{Z}} \int_{\eta_{p}-\eta_{q}}\left(f^{k}\right)^{*} \lambda=\sum_{k \in \mathbb{Z}}\left(\int_{f^{k}\left(\eta_{p}\right)} \lambda-\int_{f^{k}\left(\eta_{q}\right)} \lambda\right),
$$

where $\lambda$ is the one form (48). These sums converge without the assumption that $\lambda$ is regular because $f^{k}\left(\eta_{p, q}\right) \rightarrow A$ as $k \rightarrow \pm \infty$. Noting that $f^{k}\left(\eta_{p}\right)=g^{k}(p) \times \mathbb{S}$, and that $d y$ vanishes on the orbits of the primary intersections, the integrals above can be easily computed. For example,

$$
\int_{f^{k}\left(\eta_{p}\right)} \lambda=-S\left(g^{k-1}(p)\right) \int_{\mathbb{S}} d \theta=-2 \pi S\left(g^{k-1}(p)\right) .
$$


Hence the volume becomes

$$
\operatorname{Vol}(\overline{\mathcal{E}})=2 \pi \sum_{k \in \mathbb{Z}}\left(S\left(g^{k}(q)\right)-S\left(g^{k}(p)\right)\right) .
$$

which $2 \pi$ times the difference between the actions of the orbits of $q$ and $p$ under the symplectic map $g$. The action difference is exactly the lobe area for an area-preserving map as shown in [MMP87, Eas91, so that we have derived the obvious formula

$$
\operatorname{Vol}(\overline{\mathcal{E}})=2 \pi \operatorname{area}(\mathcal{E})
$$

As an explicit example consider the generator

$$
G(x, X)=\frac{1}{2}(X-h(x))^{2},
$$

for a diffeomorphism $h: \mathbb{R} \rightarrow \mathbb{R}$. This generator satisfies the twist condition since $\partial_{1} \partial_{2} G=$ $-h^{\prime}(x) \neq 0$ by assumption. The generated map is, explicitly, given by

$$
g(x, y)=(X, Y)=\left(h(x)+\frac{y}{h^{\prime}(x)}, \frac{y}{h^{\prime}(x)}\right) .
$$

For this case, the generator 46 is

$$
S(x, y)=\frac{1}{2}\left(\frac{y}{h^{\prime}(x)}\right)^{2}=\frac{1}{2} Y^{2} .
$$

Note that the line $y=0$ is invariant under $g$, and the dynamics on this line is simply $x \mapsto h(x)$. Thus if $h$ has exactly two fixed points, say $x_{1}<x_{2}$, then $g$ has saddle fixed points at $\left(x_{i}, 0\right)$. Two of the manifolds of these saddles simply lie on the $x$-axis; for example, if $0<h^{\prime}\left(x_{1}\right)<1<h^{\prime}\left(x_{2}\right)$ then $W^{\mathrm{s}}\left(x_{1}, 0\right)=\left(-\infty, x_{2}\right)$ and $W^{\mathrm{u}}\left(x_{2}, 0\right)=\left(x_{1}, \infty\right)$. The other two manifolds, $W^{\mathrm{u}}\left(x_{1}, 0\right)$ and $W^{\mathrm{s}}\left(x_{2}, 0\right)$, may also intersect [Tab95]. and give rise to a nontrivial resonance zone, see for example Fig. 7. For this example, there are exactly two primary intersection orbits in the upper-half plane, labelled $p$ and $q$ in the figure.

The three-dimensional map $f$ defined through (47) has an invariant plane $\{y=0\}$ and, when $h$ has two fixed points, has a pair of hyperbolic invariant circles

$$
\mathcal{C}_{i}=\left\{\left(x_{i}, 0, \theta\right): \theta \in \mathbb{S}\right\}, \quad i=1,2 .
$$

The set $\Sigma=\left\{(x, 0, \theta): x_{1}<x<x_{2}\right\}=W^{\mathrm{s}}\left(\mathcal{C}_{1}\right) \cap W^{\mathrm{u}}\left(\mathcal{C}_{2}\right)$ is a heteroclinic manifold that has zero flux. The manifolds $W^{\mathrm{u}}\left(\mathcal{C}_{1}\right)$ and $W^{\mathrm{s}}\left(\mathcal{C}_{2}\right)$ can be used to form the second, leaky boundary of a resonance zone. Indeed, if $p \in W^{\mathrm{u}}\left(\mathcal{C}_{1}\right) \cap W^{\mathrm{s}}\left(\mathcal{C}_{2}\right)$ is a primary intersection point for $g$, recall Fig. 7 , then the circle $\eta_{p}=\{p\} \times \mathbb{S}$ is a proper boundary for both $W^{\mathrm{u}}\left(\mathcal{C}_{1}\right)$ and $W^{\mathrm{s}}\left(\mathcal{C}_{2}\right)$. We can then form a resonance zone $\mathcal{R}$ with the boundary

$$
\partial \mathcal{R}=\Sigma \cup W_{f\left(\eta_{p}\right)}^{\mathrm{u}}\left(\mathcal{C}_{1}\right) \cup W_{f\left(\eta_{p}\right)}^{\mathrm{s}}\left(\mathcal{C}_{2}\right)
$$




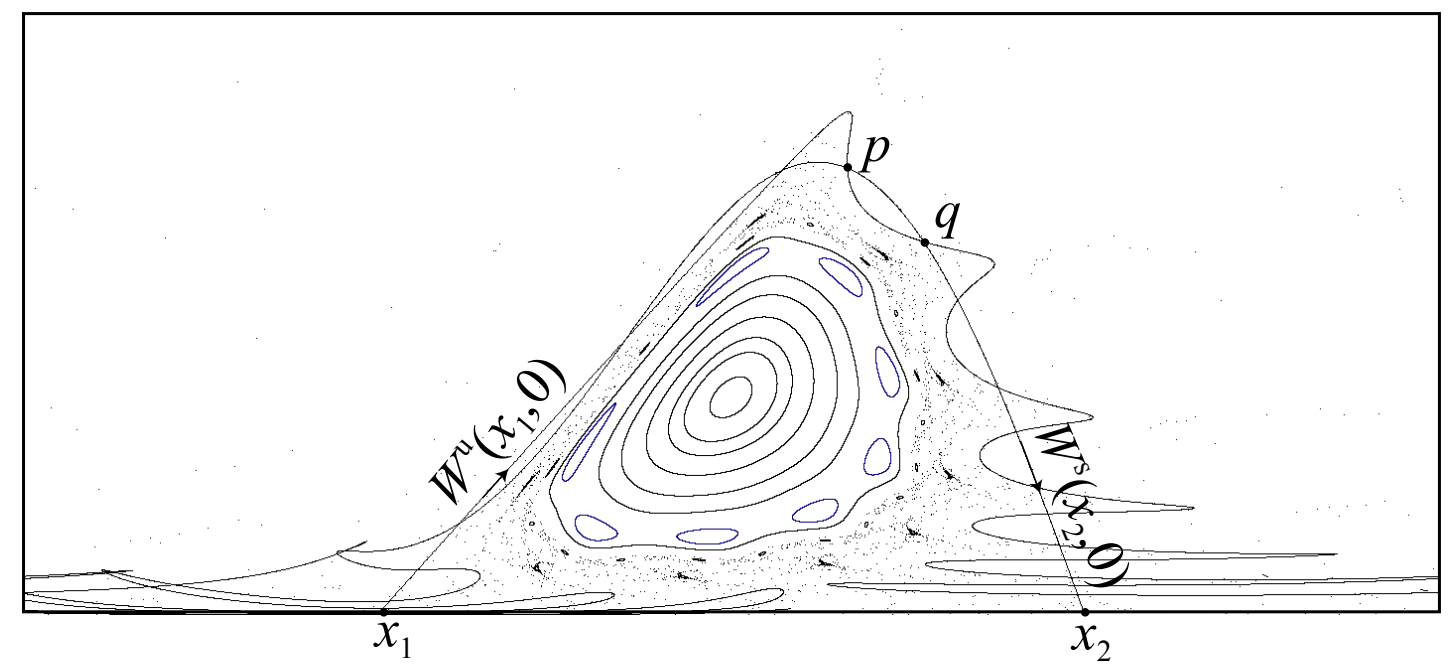

Figure 7: Phase space of the map (51) for $h(x)=x-\frac{a}{2 \pi} \cos (2 \pi x)$ with $a=0.85$. Here the saddle points lie at $x_{1}=-x_{2}=-0.25$ and there is an elliptic fixed point at $\left(0, \frac{a}{2 \pi}\right)$.

Notice that in this case, no "caps" are needed. If, as in Fig. 7, $g$ has exactly one more primary intersection point, $q$, the set of primary heteroclinic intersections between $\mathcal{C}_{1}$ and $\mathcal{C}_{2}$ is given by (49). Finally, by (50) and (52), the volume of the exit lobe is

$$
\operatorname{Vol}(\overline{\mathcal{E}})=\pi \sum_{k \in \mathbb{Z}}\left(\left(y_{k}^{q}\right)^{2}-\left(y_{k}^{p}\right)^{2}\right)
$$

\subsection{Nonautonomous Hamiltonian flow}

The flow of an incompressible vector field is volume-preserving. If the vector field is exact, in the sense we state below, then its flow will be exact as well. In this section we compute the volume of lobes that are obtained from the time- $T$ map of such a flow. For this case, our results reduce to those of [Mac94].

Recall that an incompressible vector field $X$ satisfies $L_{X} \Omega \equiv(\nabla \cdot X) \Omega=0$, where $L_{X}$ is the Lie derivative. By (64), $X$ is incompressible if the form $i_{X} \Omega$ is closed. Consequently, it is natural to say that $X$ is exact-incompressible when $i_{X} \Omega$ is exact. Indeed this implies that the flow of the vector field is exact volume-preserving.

Proposition 7 ([LM08]). Suppose $X: M \rightarrow T M$ is a vector field with complete flow $\varphi_{t}$ and $\Omega=d \alpha$ is an exact volume form on $M$. If $i_{X} \Omega$ is exact, then $L_{X} \alpha=d \beta$ for some $n-2$ form $\beta$, 
and the flow is exact volume preserving, $\varphi_{t}^{*} \alpha-\alpha=d \lambda_{t}$ with the $(n-2)$-form

$$
\lambda_{t}=\int_{0}^{t} \varphi_{\tau}^{*} \beta d \tau
$$

for each $t \in \mathbb{R}$.

As an example, consider the case of a $1+\frac{1}{2}$ degree-of-freedom Hamiltonian flow, generated by a $C^{2}$ function $H(x, y, t)$. On the extended phase space $z=(x, y, \theta) \in \mathbb{R}^{3} H$ generates the vector field

$$
X=\left(\frac{\partial H}{\partial y},-\frac{\partial H}{\partial x}, 1\right) .
$$

For the volume form $\Omega=d x \wedge d y \wedge d \theta$ we can choose $\alpha=-y d x \wedge d \theta$ so that $d \alpha=\Omega$. Hence,

$$
\begin{aligned}
& i_{X} \Omega=d H \wedge d \theta+d x \wedge d y \\
& i_{X} \alpha=-y \frac{\partial H}{\partial y} d \theta+y d x
\end{aligned}
$$

These imply

$$
L_{X} \alpha=i_{X} \Omega+d i_{X} \alpha=d\left(H-y \frac{\partial H}{\partial y}\right) \wedge d \theta,
$$

so that we can define $\beta=-\mathcal{L} d \theta$ where

$$
\mathcal{L}=y \partial_{y} H-H
$$

is the (phase space) Lagrangian. Assuming that the flow $\varphi_{t}$ of the vector field $X$ is complete, then Prop. 7 implies that it is exact volume preserving with

$$
\lambda_{t}=-\left(\int_{0}^{t} \mathcal{L} \circ \varphi_{\tau} d \tau\right) d \theta
$$

by (53). Thus $\lambda_{t}(X)$ is the (negative of the) action of the orbit segment from $\tau=0$ to $t$.

Now suppose that $H$ is 1 -periodic in its last coordinate: $H(x, y, \theta+1)=H(x, y, \theta)$, and let $M=\mathbb{R}^{2} \times \mathbb{T}$, where $\mathbb{T}=\mathbb{R} / \mathbb{Z}$.

The Poincaré return map to the section $\theta=0$ is

$$
P(x, y)=\varphi_{1}(x, y, 0)
$$

We will assume that $P$ has a saddle fixed point, $a$, whose stable and unstable manifolds intersect and have exactly two transversal, primary intersection orbits, e.g., the orbits of $p$ and $q$ as shown in Fig. 1. This implies that the vector field (54) has a hyperbolic invariant circle $A=\left\{\varphi_{t}(a, 0)\right.$ : $0 \leq t<1\}$ whose two-dimensional stable and unstable manifolds have two, primary intersection orbits, $\varphi_{t}(q)$ and $\varphi_{t}(p)$ as sketched in Fig. 8 . 


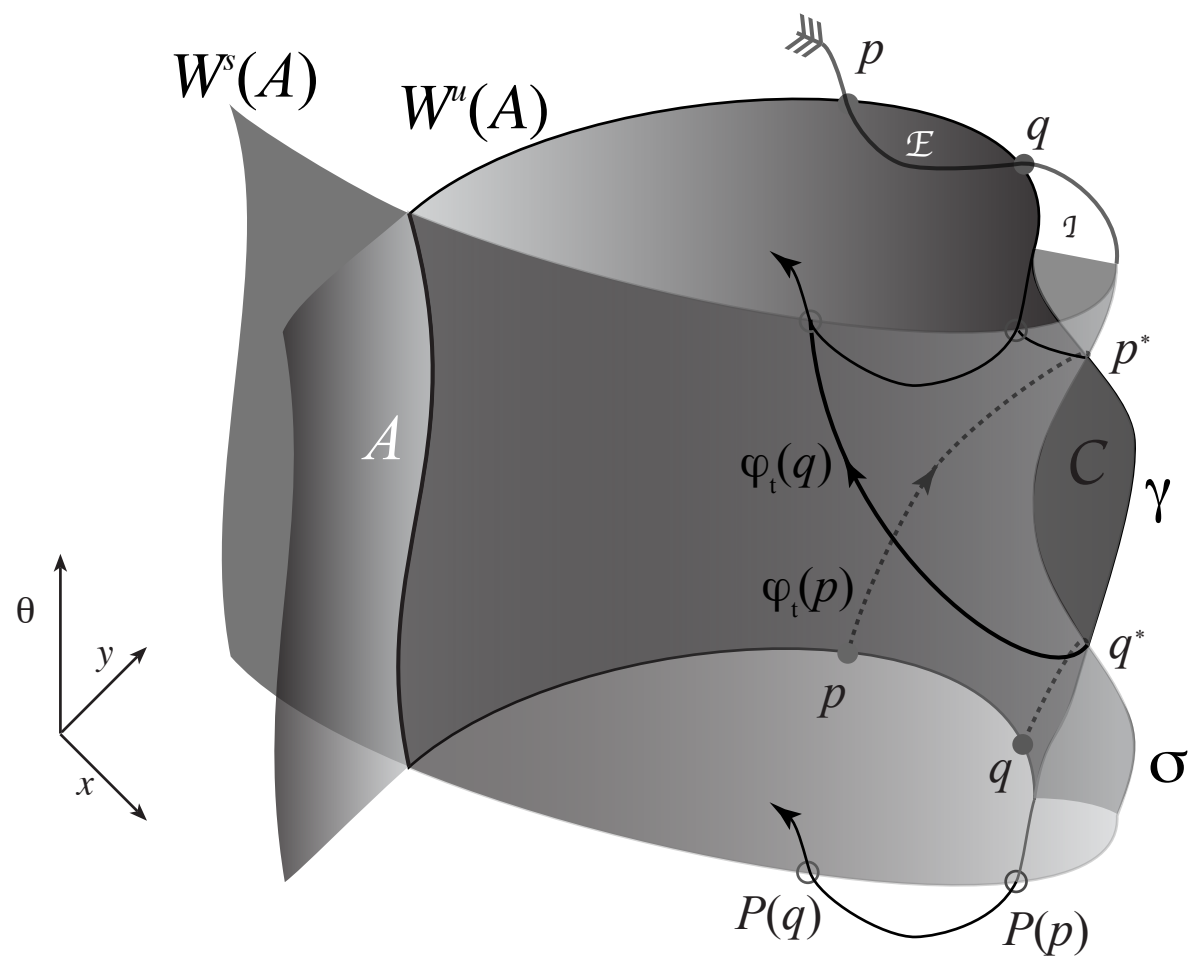

Figure 8: Resonance zone for a periodically time dependent flow with a hyperbolic invariant circle $A$ and two primary intersection orbits $\chi^{ \pm}$.

By reducing to the Poincaré section, the resonance zone and its exit and incoming sets for this three-dimensional system can be obtained purely by considering the area-preserving map $P$. In this case, we can compute the area of the exit lobe using the standard theory of [MMP84], to obtain

$$
\operatorname{area}(\mathcal{E})=\Delta W
$$

where $\Delta W$ is the difference between the actions of the two primary homoclinic orbits:

$$
\Delta W=\int_{-\infty}^{\infty}\left(\mathcal{L}\left(\varphi_{\tau}(q)\right)-\mathcal{L}\left(\varphi_{\tau}(p)\right)\right) d \tau
$$

Note that since $P$ is the time-one map of $X$, area $(\mathcal{E})$ is the area per unit time that escapes from the resonance zone.

To make a test case for Thm. 4, we now consider the three-dimensional map

$$
f(x, y, \theta) \equiv \varphi_{T}(x, y, \theta)
$$

for $T \notin \mathbb{Z}$. By Prop. 7, $f$ is an exact volume-preserving diffeomorphism of $M$ with one form $\lambda_{T}$ given by (55). Under the above assumptions, $A$ is a normally hyperbolic invariant circle of the map $f$, and $f$ satisfies (H1)-(H3). 
To construct a resonance zone, $\overline{\mathcal{R}}$, we must introduce caps: since the orbits of $p$ and $q$ are homotopic to $A$, they cross any proper loops $\sigma$ and $\gamma$. As usual we select a pair of proper loops $\gamma$ and $\sigma$, their corresponding fundamental domains $\mathcal{U}$ and $\mathcal{S}$, and a cap $\mathcal{C}$ obeying (H4)-(H6). We let $\overline{\mathcal{E}}$ denote the three-dimensional exit lobe for this system. Using the form $(55)$, since $\varphi_{t}^{*} d \theta=d \theta$ we have

$$
\left(f^{k}\right)^{*} \lambda_{T}=-\left(\varphi_{k T}\right)^{*}\left(\int_{0}^{T} \mathcal{L} \circ \varphi_{\tau} d \tau\right) d \theta=-\left(\int_{k T}^{(k+1) T} \mathcal{L} \circ \varphi_{\tau} d \tau\right) d \theta
$$

The set $\eta \subset \mathcal{P}(A, A)$ used in Thm. 4 corresponds to the primary intersections on the fundamental domains, thus, $\eta=\eta_{p}-\eta_{q}$ where

$$
\begin{aligned}
& \eta_{p}=\left\{\varphi_{t}(p): t \in \mathbb{R}\right\} \cap \mathcal{U}=\left\{\varphi_{s}\left(p^{*}\right): 0 \leq s \leq T\right\} \\
& \eta_{q}=\left\{\varphi_{t}(q): t \in \mathbb{R}\right\} \cap \mathcal{U}=\left\{\varphi_{s}\left(q^{*}\right): 0 \leq s \leq T\right\}
\end{aligned}
$$

and $\left\{p^{*}, q^{*}\right\}=\gamma \cap \sigma$ are points on the orbits of $p$ and $q$.

Theorem 4 implies that, in order to compute the volume, we must integrate the one form given in (58) over $\eta$ and sum over $k \in \mathbb{Z}$. Using the temporal parametrization for $\eta_{p}$ gives $\left.d \theta\right|_{\eta_{p}}=d s$, and

$$
\int_{\eta_{p}}\left(f^{k}\right)^{*} \lambda_{T}=-\int_{0}^{T}\left(\int_{k T}^{(k+1) T} \mathcal{L}\left(\varphi_{t+s}\left(p^{*}\right)\right) d t\right) d s=-T \int_{k T}^{(k+1) T} \mathcal{L}\left(\varphi_{\tau}\left(p^{*}\right)\right) d \tau,
$$

where we defined a new integration variable $\tau=t+s$ and used the periodicity of $\mathcal{L}$ in time. The volume of the exit lobe for $f$ is given by (21), and the sum becomes a single integral:

$$
\operatorname{Vol}(\overline{\mathcal{E}})=-T \int_{-\infty}^{\infty}\left(\mathcal{L}\left(\varphi_{t}\left(p^{*}\right)\right)-\mathcal{L}\left(\varphi_{t}\left(q^{*}\right)\right)\right) d t
$$

This integral is geometrically convergent since the orbits of $p^{*}$ and $q^{*}$ are bi-asymptotic to the hyperbolic circle $A$. Moreover, we can replace the initial conditions with $p$, and $q$, and thus obtain

$$
\operatorname{Vol}(\overline{\mathcal{E}})=T \Delta W
$$

where $\Delta W$ is given by $(57)$. Note that $\operatorname{Vol}(\overline{\mathcal{E}})$ is the volume that exits from the three-dimensional resonance per step of the map $f$, that is per $T$ units of time. Thus, (59) is exactly what is expected from (56).

\section{Conclusion and future research}

The computation of lobe volumes is a first step toward developing a theory of transport for volumepreserving maps. These maps appear to have all sticky regions surrounding invariant tori and algebraic decay of exit and transit time distributions, complications that are familiar from the study of area-preserving maps [Mei92]. Since volume-preserving dynamics pertains to the motion 
of Lagrangian tracers in incompressible fluids, a theory of transport should prove useful for the understanding laminar mixing and for designing an optimal mixer [Bal05].

To apply our result (21) to a general map we must compute the primary intersection $\mathcal{P}(A, B)$ between a pair of invariant sets $A$ and $B$. Finding this set is easiest when the map $f$ is reversible, $R \circ f=f^{-1} \circ R$, and when $R(A)=B$ for the reversor $R$. In this case, it is easy to see that if the manifold $W^{\mathrm{s}}(A)$ intersects the fixed set of the reversor, $\operatorname{Fix}(R)$, the intersection point must be a point in the primary intersection set $\mathcal{P}$. Thus this point can be used as a starting point for a continuation method to obtain $\eta$. The intersection with $\operatorname{Fix}(f \circ R)$ will give a point on a second component of $\eta$. An suitable example to study is the quadratic map of [LM98], which is reversible in special cases. We hope to report such computations in a future paper.

\section{A Some Notation}

Here we set out our notation, which follows e.g. [AM78]. If $\alpha$ is a $k$-form and $V_{1}, V_{2}, \ldots V_{k}$ are vector fields, then the pullback, $f^{*}$, of a diffeomorphism $f$ is defined by

$$
\left(f^{*} \alpha\right)_{x}\left(V_{1}, V_{2}, \ldots, V_{k}\right)=\alpha_{f(x)}\left(D f(x) V_{1}(x), \ldots, D f(x) V_{k}(x)\right) .
$$

The pullback can be applied to a vector field $V$ as well:

$$
\left(f^{*} V\right)(x)=(D f(x))^{-1} V(f(x))
$$

The pushforward operator is defined as

$$
f_{*}=\left(f^{-1}\right)^{*} .
$$

The inner product of $\alpha$ with $V$ is defined as the $(k-1)$-form

$$
i_{V} \alpha=\alpha(V, \cdot, \ldots, \cdot)
$$

Suppose that $\varphi_{t}$ is the $\left(C^{1}\right)$ flow of a vector field $V$, so that $\varphi_{0}(x)=x$, and $d / d t \varphi_{t}(x)=V\left(\varphi_{t}(x)\right)$. Then the Lie derivative with respect to $V$ is the differential operator defined by

$$
\left.L_{V} \cdot \equiv \frac{d}{d t}\right|_{t=0} \varphi_{t}^{*}
$$

where $\cdot$ is any tensor. The key identity for the derivative is Cartan's magic formula:

$$
L_{V} \equiv i_{V}(d)+d\left(i_{V}\right)
$$




\section{References}

\section{References}

[AM78] R. Abraham and J.E. Marsden. Foundations of Mechanics. Benjamin Cummings, 1978.

[Bal05] S. Balasuriya. Optimal perturbation for enhanced chaotic transport. Phys. D, 202(34):155-176, 2005.

[Bro81] H.W. Broer. Formal normal form theorems for vector fields and some consequences for bifurcations in the volume preserving case. In Dynamical systems and turbulence, Warwick 1980 (Coventry, 1979/1980), volume 898, pages 54-74. Springer-Verlag, Berlin, 1981.

[Car04] M.M. Carroll. A representation theorem for volume-preserving transformations. Int. J. of Non-Linear Mech., 39:219-224, 2004.

[CFdlL05] X. Cabré, E. Fontich, and R. de la Llave. The parameterization method for invariant manifolds. III. Overview and applications. J. Differential Equations, 218(2):444-515, 2005 .

[DdlLS08] A. Delshams, R. de la Llave, and T.M. Seara. Geometric properties of the scattering map of a normally hyperbolic invariant manifold. Adv. Math., 217(3):1096-1153, 2008.

[Eas91] R.W. Easton. Transport through chaos. Nonlinearity, 4:583-590, 1991.

[Fro72] C. Froeschlé. Numerical study of a four dimensional mapping. Astron. and Astrophys, 16:172-189, 1972.

[GM03] G. Gaeta and P. Morando. A variational principle for volume-preserving dynamics. $J$. Nonlinear Math. Phys., 10(4):539-554, 2003.

[HM82] P.J. Holmes and J.E. Marsden. Melnikov's method and Arnold diffusion for perturbations of integrable Hamiltonian systems. J. Math. Phys., 23(4):669-675, 1982.

[Hol84] P.J. Holmes. Some remarks on chaotic particle paths in time-periodic, three-dimensional swirling flows. In Fluids and plasmas: geometry and dynamics (Boulder, Colo., 1983), volume 28 of Contemp. Math., pages 393-404. Amer. Math. Soc., 1984.

[HPS77a] M.W. Hirsch, C.C. Pugh, and M. Shub. Invariant manifolds. Lecture Notes in Mathematics, Vol. 583. Springer-Verlag, Berlin, 1977.

[HPS77b] M.W. Hirsch, C.C. Pugh, and M. Shub. Invariant manifolds, volume 583 of Lecture notes in mathematics. Springer-Verlag, New York, 1977. 
[LM98] H.E. Lomelí and J.D. Meiss. Quadratic volume-preserving maps. Nonlinearity, 11:557$574,1998$.

[LM00] H.E. Lomelí and J.D. Meiss. Heteroclinic primary intersections and codimension one Melnikov method for volume-preserving maps. Chaos, 10(1):109-121, 2000.

[LM03] H.E. Lomelí and J.D. Meiss. Heteroclinic intersections between invariant circles of volume-preserving maps. Nonlinearity, 16(5):1573-1595, 2003.

[LM08] H.E. Lomelí and J.D. Meiss. Generating forms for exact volume-preserving maps. Technical report, University of Colorado, 2008.

[LMRR08] H.E. Lomelí, J.D. Meiss, and R. Ramírez-Ros. Canonical Melnikov theory for diffeomorphisms. Nonlinearity, 21:485-508, 2008.

[LRR08] H.E. Lomelí and R. Ramírez-Ros. Separatrix splitting in 3D volume-preserving maps. SIAM J. Appl. Dyn. Sys., in press, 2008.

[Mac94] R.S. MacKay. Transport in 3D volume-preserving flows. J. Nonlin. Sci, 4:329-354, 1994.

[Mei92] J.D. Meiss. Symplectic maps, variational principles, and transport. Rev. Mod. Phys., 64(3):795-848, 1992.

[Mei97] J.D. Meiss. Average exit time for volume preserving maps. Chaos, 7:139-147, 1997.

[Mel63] V.K. Melnikov. On the stability of the center for time periodic perturbations. Trans. Moscow Math. Soc., 12:1-57, 1963.

[MM88] R.S. MacKay and J.D. Meiss. Relationship between quantum and classical thresholds for multiphoton ionization of excited atoms. Phys. Rev. A, 37(12):4702-4706, 1988.

[MMP84] R.S. MacKay, J.D. Meiss, and I.C. Percival. Transport in Hamiltonian systems. Physica $D, 13: 55-81,1984$.

[MMP87] R.S. MacKay, J.D. Meiss, and I.C. Percival. Resonances in area-preserving maps. Physica $D, 27: 1-20,1987$.

[RKW88] V. Rom-Kedar and S. Wiggins. Transport in two-dimensional maps. Arc. Rational Mech. Anal., 109(3):239-298, 1988.

[Tab95] E. Tabacman. Variational computation of homoclinic orbits for twist maps. Phys. D, 85(4):548-562, 1995. 
[Wig92] S. Wiggins. Chaotic Transport in Dynamical Systems. Springer-Verlag, 1992.

[Xia92] Z. Xia. Existence of invariant tori in volume-preserving diffeomorphisms. Erg. Th. Dyn. Sys., 12(3):621-631, 1992. 